\title{
Striatopallidal dysfunction underlies repetitive behavior in Shank3-deficient model of autism
}

\author{
Wenting Wang, ${ }^{1,2}$ Chenchen Li, ${ }^{2,3}$ Qian Chen, ${ }^{2}$ Marie-Sophie van der Goes, ${ }^{2}$ James Hawrot, ${ }^{2,3}$ Annie Y. Yao, ${ }^{2,3}$ Xian Gao, ${ }^{2,4}$ \\ Congyi Lu, ${ }^{2}$ Ying Zang, ${ }^{3,5}$ Qiangge Zhang, ${ }^{2}$ Katherine Lyman, ${ }^{3}$ Dongqing Wang, ${ }^{2}$ Baolin Guo, ${ }^{1}$ Shengxi Wu, ${ }^{1}$ Charles R. Gerfen, ${ }^{6}$ \\ Zhanyan $\mathrm{Fu}^{2,3}$ and Guoping Feng ${ }^{2,3}$ \\ 'Department of Neurobiology and Collaborative Innovation Center for Brain Science, Institute of Neuroscience, School of Basic Medicine, Fourth Military Medical University, Xi'an, Shanxi, China. ${ }^{2}$ McCovern \\ Institute for Brain Research, Department of Brain and Cognitive Sciences, Massachusetts Institute of Technology (MIT), Cambridge, Massachusetts, USA. ${ }^{3 S}$ tanley Center for Psychiatric Research, Broad \\ Institute of MIT and Harvard, Cambridge, Massachusetts, USA. ${ }^{4}$ Key Laboratory of Brain Functional Genomics (Ministry of Education and Science and Technology Commission of Shanghai Municipality), \\ Institute of Cognitive Neuroscience, School of Psychology and Cognitive Science, East China Normal University, Shanghai, China. ${ }^{5}$ Pain Research Center and Department of Physiology, Zhongshan Medical \\ School, Sun Yat-Sen University, Guangzhou, Guang Dong, China. ' Laboratory of System Neuroscience, National Institute of Mental Health (NIMH), Bethesda, Maryland, USA.
}

\begin{abstract}
The postsynaptic scaffolding protein SH3 and multiple ankyrin repeat domains 3 (SHANK3) is critical for the development and function of glutamatergic synapses. Disruption of the SHANK3-encoding gene has been strongly implicated as a monogenic cause of autism, and Shank3 mutant mice show repetitive grooming and social interaction deficits. Although basal ganglia dysfunction has been proposed to underlie repetitive behaviors, few studies have provided direct evidence to support this notion and the exact cellular mechanisms remain largely unknown. Here, we utilized the Shank3B mutant mouse model of autism to investigate how Shank3 mutation may differentially affect striatonigral (direct pathway) and striatopallidal (indirect pathway) medium spiny neurons (MSNs) and its relevance to repetitive grooming behavior in Shank3B mutant mice. We found that Shank3 deletion preferentially affects synapses onto striatopallidal MSNs. Striatopallidal MSNs showed profound defects, including alterations in synaptic transmission, synaptic plasticity, and spine density. Importantly, the repetitive grooming behavior was rescued by selectively enhancing the striatopallidal MSN activity via a Gq-coupled human M3 muscarinic receptor (hM3Dq), a type of designer receptors exclusively activated by designer drugs (DREADD). Our findings directly demonstrate the existence of distinct changes between 2 striatal pathways in a mouse model of autism and indicate that the indirect striatal pathway disruption might play a causative role in repetitive behavior of Shank3B mutant mice.
\end{abstract}

\section{Introduction}

Recent genetic studies have revealed a large number of risk genes for autism spectrum disorder (ASD). Many of these genes encode proteins important for synaptic development and function. One such gene is $\mathrm{SH} 3$ and multiple ankyrin repeat domains 3 (SHANK3). SHANK3 is a member of the Shank family of proteins (SHANK1-3). SHANK proteins interact with various postsynaptic density (PSD) proteins through 5 main functional domains: $\mathrm{N}$-terminal ankyrin repeats, $\mathrm{SH} 3$ domain, PDZ domain, prolinerich region, and a C-terminal sterile $\alpha$ motif (SAM) (1-5). Most notably, SHANKs bind to synapse-associated protein 90/postsynaptic density 95-associated protein (SAPAP, also known as GKAP or DLGAP), which in turn binds to PSD95 family proteins to form the PSD95/SAPAP/SHANK postsynaptic complex $(2,4$, $6,7)$. These 3 groups of multidomain proteins are proposed as

\section{Related Commentary: p. 1618}

Authorship note: W. Wang, C. Li, and Q. Chen contributed equally to this study. Conflict of interest: The authors have declared that no conflict of interest exists. Submitted: April 11, 2016; Accepted: February 16, 2017. Reference information: / Clin Invest. 2017;127(5):1978-1990. https://doi.org/10.1172/JCI87997. forming a key postsynaptic scaffold, playing an important role in targeting, anchoring, and dynamically regulating synaptic localization of neurotransmitter receptors and signaling molecules (8). SHANKs are also connected to the metabotropic glutamate receptor (mGluR) pathway through binding to Homer (9-11). In addition, given the SHANK protein's link to actin-binding proteins, SHANKs have been shown to regulate spine development (10, $12,13)$. Thus, SHANK proteins have been proposed as playing key roles in synaptic development and function $(11,14,15)$.

Disruption of SHANK3 is thought to be the cause of the core neurodevelopmental and neurobehavioral deficits in PhelanMcDermid syndrome (PMS, 22q13.3 deletion syndrome), as individuals carrying a ring chromosome 22 with an intact SHANK3 gene are phenotypically normal (16-22). PMS is a neurodevelopmental disorder characterized by intellectual disability, autisticlike behaviors, hypotonia, and impaired development of speech and language $(23,24)$. Moreover, recent genetic studies have identified a large number of SHANK3 mutations in ASD patients not diagnosed with PMS (20, 25-28), strongly implicating SHANK3 gene disruption or mutation as a monogenic cause of ASD $(18,20$, $23,29,30)$. In support of these genetic findings, Shank3 mutant mice exhibited various synaptic defects and ASD-related behavioral abnormalities, including repetitive grooming behaviors and 
A D1 MSN
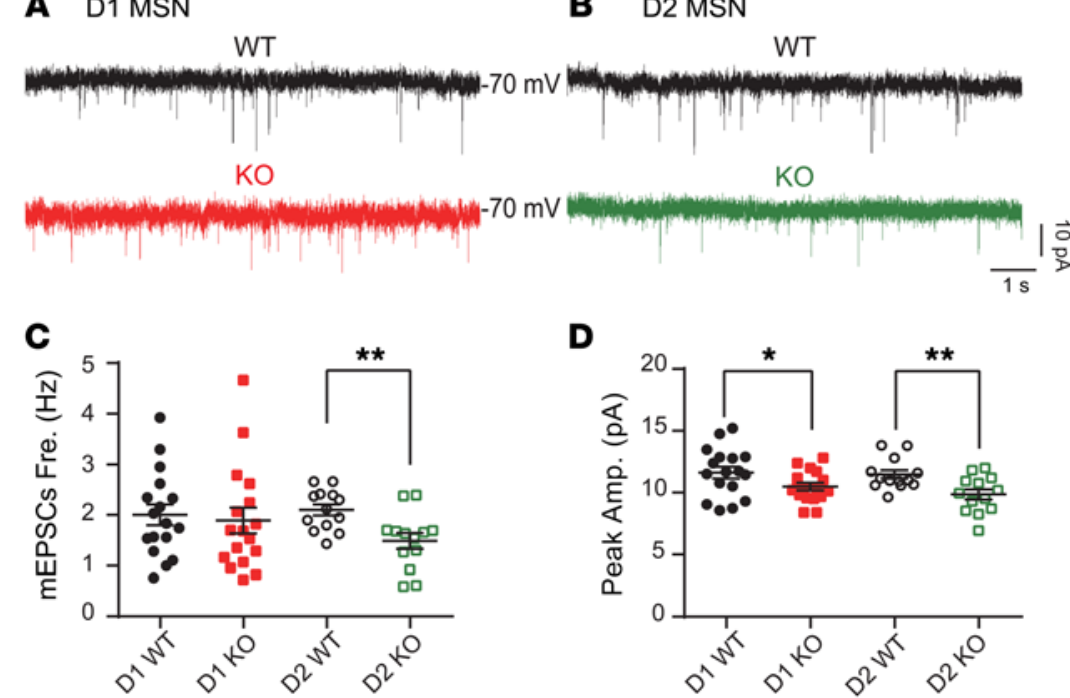

$\mathbf{E}$

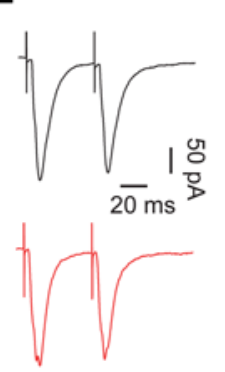

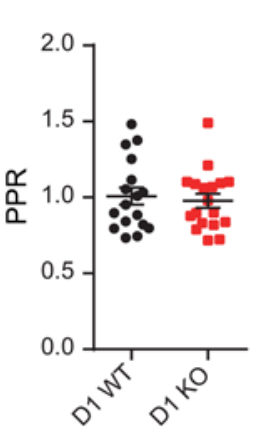

D

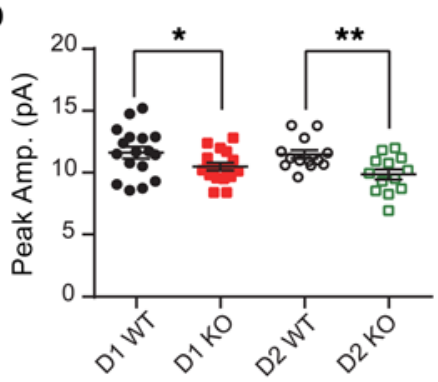

$\mathbf{F}$

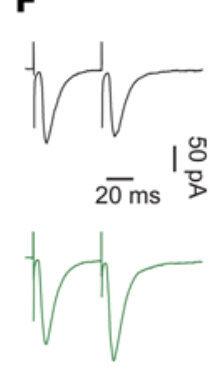

Figure 1. Differential effects of Shank3 deletion on glutamatergic synaptic transmission in D1 and D2 MSNs. (A and $\mathbf{B})$ Representative recording traces of AMPA-mediated mEPSCs of D1 MSNs (A) and D2 MSNs (B) in the presence of TTX $(0.5 \mu \mathrm{M})$, PTX $(100 \mu \mathrm{M})$, and DL-APV $(50 \mu \mathrm{M})$ from acute striatal brain slices. (C and D) Summary data of mEPSC frequency (C) and peak amplitude (pA) (D) from D1 MSNs and D2 MSNs of WT and KO mice (mEPSC frequency: WT D1, $n=17, N=4, \mathrm{KO} D 1, n=17, N=4, P=0.73$. WT D2, $n=13, N=3$; KO D2, $n=13, N=3, P=0.0039$; mEPSC amplitude: WT D1 and KO D1, $P=0.053$. WT D2 and KO D2, $P=0.006$ ). (E) Representative traces and summary data of PPR of D1 MSNs from WT and KO mice. (F) Representative traces and summary data of PPR of D2 MSNs from WT and KO mice (PPR: WT D1, $n=17, N=4$, KO D1, $n=18, N=4, P=0.67$; WT D2, $n=15, N=4$, KO D2, $n=15, N=4, P=0.048$ ). An unpaired $t$ test was used for comparisons. ${ }^{*} P<0.05$; ${ }^{* *} P<0.01$. $n$, cell number; $N$, animal number. social interaction deficits (31-36). Thus, Shank3 mutant mice provide us with a monogenic model for studying neural mechanisms underlying autism-relevant behaviors.

Dysfunction of the basal ganglia has been strongly implicated in a variety of psychiatric disorders characterized by inflexible and maladaptive behaviors, such as obsessive and compulsive disorders (OCD), drug addiction, Tourette syndrome, and ASD (37-41). The dorsal striatum, the largest nucleus of the basal ganglia, receives converging information from cortex and thalamus to mainly control voluntary motor actions and procedural learning $(42,43)$. Output from the dorsal striatum is organized into 2 primary projection pathways, dopamine D1 receptor expressing striatonigral neurons forming the direct projection pathway (D1 MSN, where MSN indicates medium spiny neuron) and D2 receptor expressing striatopallidal neurons forming the indirect striatal projection pathway (D2 MSN), respectively $(44,45)$. Instead of the opposing roles of the 2 pathways suggested in the classical model of basal ganglia circuitry, recent findings highlight that the cooperative, concurrent, and balanced activity of the 2 pathways is critical for motor control, action initiation, and selection (46-48). Our previous studies demonstrated that disruption of the Shank3 gene in mice resulted in both structural and functional defects in excitatory synapses of MSNs in the dorsal striatum $(31,49,50)$. Although these striatal synaptic defects have been proposed to be the cause of repetitive and compulsive grooming behavior in Shank3B mutant mice, direct evidence to support this hypothe- sis is still lacking. In particular, it is not clear whether the direct and indirect pathways of the basal ganglia are differentially affected in Shank3B mutant mice.

For the current study, we performed a systematic characterization of synaptic transmission, synaptic plasticity, neuronal morphology in D1 and D2 MSNs as well as their role in repetitive behaviors in the Shank3B mutant mouse model of ASD. We revealed both distinct and shared defects in these 2 primary subtypes of striatal neurons: D2 MSNs showed marked defects across all parameters examined, including pre- and postsynaptic synaptic function, synaptic plasticity, and spine density, while D1 MSNs only presented postsynaptic defects with a reduced $\alpha$-amino-3-hydroxy-5-methyl-4-isoxazolepropionic acid receptor (AMPAR) response. Consistent with the more profound synaptic defects in D2 MSNs in Shank3B mutant mice, the repetitive grooming behavior was corrected by selectively enhancing indirect pathway activity, but not direct pathway, activity. Our findings provide direct evidence of distinct changes between the 2 striatal pathways in a mouse model of autism and highlight that indirect striatal pathway disruption might play a causative role in repetitive behavior.

\section{Results}

Shank3 deletion preferentially affects glutamatergic synaptic transmission in striatopallidal MSNs. To specifically test whether deletion of the Shank3 gene differentially affects synapses of D1 MSN and D2 MSNs, we crossed Shank3B-KO mice with transgenic mice 
that expressed either tdTomato in D1R-expressing direct pathway-projecting MSNs (Drd1a-tdTomato mice) (51) or EGFP in D2R-expressing indirect pathway-projecting MSNs (Drd2-EGFP mice) (52). Using whole-cell voltage clamp recordings, we measured AMPAR-mediated miniature excitatory postsynaptic currents (mEPSCs) in the presence of tetrodotoxin (TTX) and picrotoxin (PTX). We found a reduction of mEPSC peak amplitude in both D1 MSNs and D2 MSNs of Shank3B-KO mice. However, the frequency of AMPAR-mediated mEPSCs was reduced only in D2 MSNs, but not D1 MSNs (Figure 1, A-D). Such distinct glutamatergic synaptic transmission changes in D2 MSNs suggest that Shank3 loss may lead to a reduced number of synapses, reduced presynaptic release probability, or both selectively onto D2 MSNs, while leaving D1 MSNs relatively intact. We thus measured pairedpulse ratio (PPR, $50 \mathrm{~ms}$ of interpulse interval) to assess presynaptic release probability. A significant enhancement in PPR was found only in D2 MSN recordings in Shank3B-KO mice, indicative of reduced presynaptic release probabilities of the glutamatergic presynaptic terminals innervating D2 MSNs in Shank3B-KO mice (Figure 1, E and F). The selective alteration in presynaptic release probability likely contributes, at least partially, to the reduced frequency of mEPSCs observed in D2 MSNs in Shank3B-KO mice.

Another important component that regulates striatal MSN activity is the local GABAergic interneuron. Electrophysiological studies have demonstrated that striatal MSNs receive prominent GABAergic innervations. Moreover, these striatal GABAergic interneurons have distinct firing patterns and connections with MSNs and thus may exert different effects on D1 and D2 MSNs $(53,54)$. As shown in Supplemental Figure 1 (supplemental material available online with this article; https://doi.org/10.1172/ JCI87997DS1), we did not observe significant changes in both frequency and peak amplitude of GABA $_{\mathrm{A}}$ receptor-mediated miniature inhibitory postsynaptic currents (mIPSCs) in D1 and D2 MSNs of Shank3B-KO mice. In addition, the final output activity of MSNs is also determined by intrinsic membrane properties; we thus examined and compared the MSN membrane properties between Shank3B-KO and WT mice. As shown in Supplemental Table 1, we did not observe significant alterations in resting membrane potential, threshold potential, membrane input resistance, or rheobase amplitude between Shank3B-KO and WT mice. However, when we examined the frequency-current (F/I) relationship, we found a left shift trend of the averaged F/I curve in D2 MSNs of Shank3B-KO mice as compared with D2 MSNs of WT mice. Although 2-way ANOVA interaction analysis showed no statistical significance (Supplemental Figure 2) using Sidak's multiple comparisons test, the firing frequency of D2 MSNs in Shank3B-KO mice was significantly higher than in WT D2 MSNs, suggesting an increased intrinsic excitability of Shank3B-KO D2 MSNs.

Shank3 deletion selectively impaired endocannabinoid-mediated long-term depression in D2 MSNs. Long-lasting changes in synaptic strength at MSN glutamatergic synapses have been implicated in habitual learning and striatal-based cognitive functions. The plasticity of the MSN glutamatergic synapses is known to be modulated by endocannabinoid (eCB), and eCB-dependent long-term depression (eCB-LTD) is one of the most dominant forms of long-term plasticity expressed at MSN glutamatergic synapses (55-58). eCBs are released by postsynaptic neurons and act as retrograde signaling to activate presynaptically located $\mathrm{CB} 1$ receptors (CB1Rs) to reduce neurotransmission. However, there is controversy in the literature as to whether the eCB-LTD can be induced at glutamatergic synapses onto both D1 and D2 MSNs because high-frequency electrical stimulation (eHFS) might recruit intrastriatal dopaminergic and cholinergic signaling that can modulate this form of LTD (56-60). We thus started out to test whether the induction protocol, pairing HFS (4 trains of 100 $\mathrm{Hz} / \mathrm{s}$ given at 10-second interval) of presynaptic glutamatergic afferent fibers by placing a stimulating electrode at the inner edge of the corpus callosum (instead of intrastriatal stimulation) and postsynaptic depolarization (DP) of MSNs (HFS/DP), was able to induce LTD in both types of MSNs and whether LTD could be blocked by a CB1R antagonist in both types of neurons. Consistent with previous reports $(55,56,58,61-63)$, the HFS/DP stimulation paradigm led to the induction of robust LTD in WT D1 MSNs and D2 MSNs in the dorsal striatum, as evidenced by the reduction in the amplitude of evoked EPSCs (eEPSCs) (Figure 2). This reduction in eEPSC amplitude following LTD induction was also accompanied by an increase in PPR, which suggests a reduction in presynaptic glutamate release probability. In addition, as shown in Supplemental Figure 3, the LTD in both types of neurons can be blocked by AM251, a CB1R antagonist, suggesting that the LTD at both D1 and D2 MSN synapses was mediated by the retrograde signaling eCB. As shown in Figure 2, A-D, both the reduction in the amplitude of eEPSCs and the increase in PPR in D1 MSNs following LTD induction are comparable between WT and Shank3BKO mice. However, in response to the same HFS/DP stimulation paradigm, the activity-dependent reduction of eEPSC amplitude in Shank3B-KO D2 MSNs was significantly less when compared with D2 MSNs in WT mice (Figure 2, E-G, eEPSC amplitude in the last 10 minutes relative to baseline). Consistently, the increase in PPR (50 ms of interpulse interval) of eEPSCs following the highfrequency stimulation of glutamatergic afferents was not observed in D2 MSNs from Shank3B-KO mice (PPR in the last 10 minutes relative to baseline, Figure $2 \mathrm{H}$ ). Together, these data demonstrate that the loss of Shank3 selectively impaired LTD in D2 MSNs.

The HFS/DP striatal LTD induction requires the coactivation of postsynaptic Cav1.3 L-type calcium channels and the predominant group I mGluRs in striatum, type 5 metabotropic glutamate receptors (mGluR5) in MSNs, which leads to a robust elevation in dendritic $\mathrm{Ca}^{2+}$ levels $(55,57,64,65)$. This activity-induced elevation in dendritic $\mathrm{Ca}^{2+}$ levels then triggers the production of eCB, which acts on presynaptic CB1Rs. The CB1R, a Gi/o-coupled receptor, is known to inhibit glutamate release when activated. Thus, striatal LTD induction originates from postsynaptic sites, but expresses through a presynaptic mechanism involving a decrease in glutamate release probability via retrograde eCB signaling. We thus tested whether Shank3 disruption specifically affected mGluR5 activity and Cav1.3 activity, thereby contributing to impaired HFS/DP-LTD in D2 MSNs.

We first induced corticostriatal eCB-LTD by pharmacological activation of group $1 \mathrm{mGluRs}$ (mGluR1 and mGluR5) using the selective agonist (R,S)-3,5-dihydroxyphenylglycine (DHPG). We observed a robust induction of LTD in WT D2 MSNs following the application of $100 \mu \mathrm{M}$ DHPG for 10 to 15 minutes in the presence of NMDA receptor (NMDAR) blocker DL-2-amino-5-phospho- 
D1 MSNs
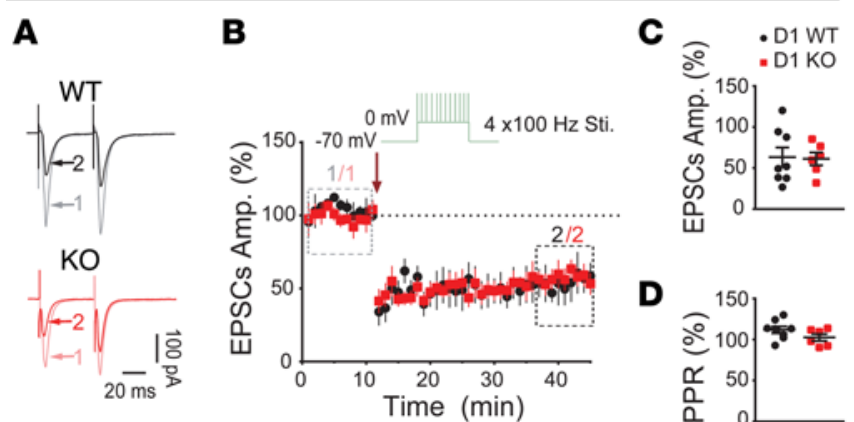

J

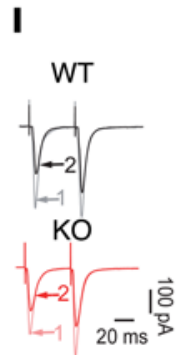

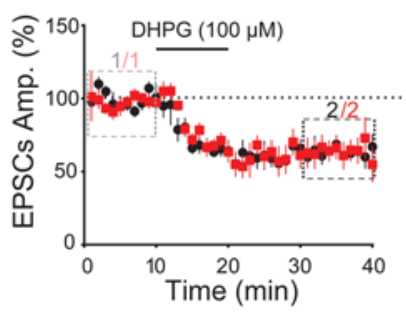

K

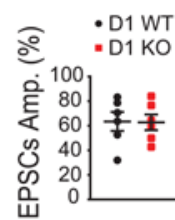

L

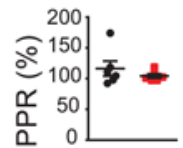

D2 MSNs
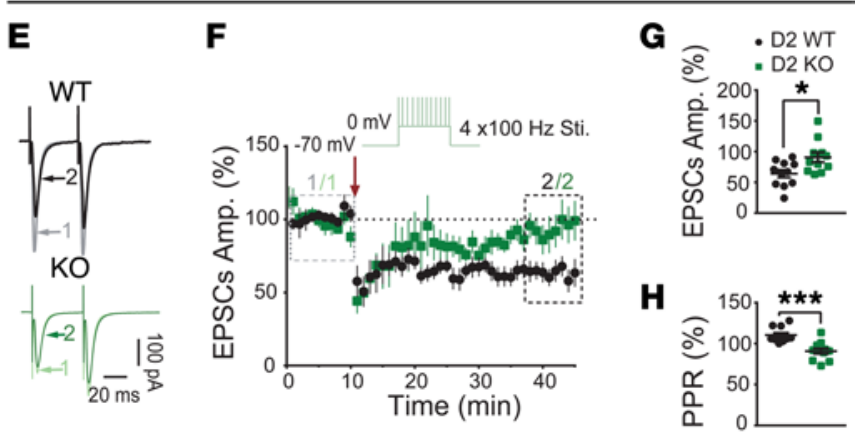

M

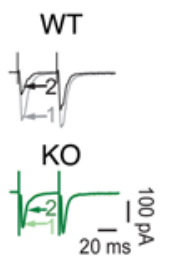

$\mathbf{N}$

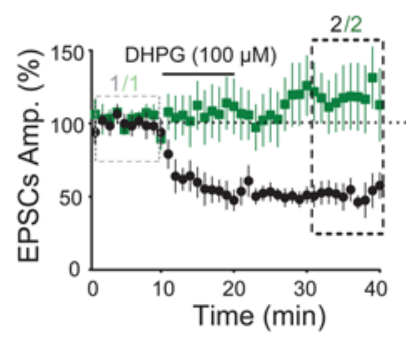

0
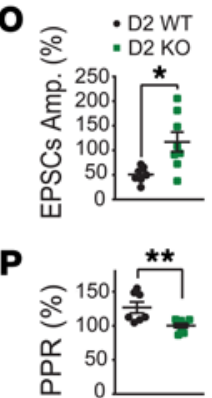

Figure 2. Shank3 deletion selectively impaired LTD in D2 MSNs, but not D1 MSNs. (A, I, E, M) Averaged traces from a representative LTD experiment in D1 (A and $\mathbf{I})$ and D2 MSNs (E and $\mathbf{M}$ ) using HFS-LTD protocol (A and $\mathbf{E}$ ) and DHPG-LTD protocol (I and $\mathbf{M})$. Light traces represent the baseline EPSC average from 0 to 10 minutes (labeled with light color number 1), and dark traces represent the average EPSC from the last 10 minutes after LTD induction (dark color number 2). (B, J, F, N) LTD is similar between WT and KO D1 MSNs in both HFS-LTD (B) and DHPG-LTD (J). KO D2 MSNs show less reduction level compared with WT D2 MSNs in both HFS-LTD (F) and DHPG-LTD (N). (C, G, K, O) Summary data of eEPSC amplitudes of D1 MSNs (C and K) and D2 MSNs ( $\mathbf{G}$ and $\mathbf{O}$ ) from WT and KO mice using the HFS-LTD protocol ( $\mathbf{C}$ and $\mathbf{G}$ ) and the DHPG-LTD protocol (K and $\mathbf{0})$ (comparison between baseline and the last 10 minutes of recording). C, D1 MSNs, WT, $n=8, N=3$; KO, $n=6, N=3, P=0.89 ; \mathbf{G}$, D2 MSNs: WT, $n=11, N=6$; KO, $n=11, N=6, P=0.0165 ; \mathrm{K}, \mathrm{D} 1 \mathrm{MSNs}$ : WT, $n=6, N=2 ; \mathrm{KO}, n=6, N=2, P=0.95: \mathbf{0}$, D2 MSNs: WT, $n=7, N=3 ; \mathrm{KO}, n=8, N=3, P=0.0104$. (D, H, L, P) Summary data of PPR of D1 MSNs (D and L) and D2 MSNs (H and $\mathbf{P})$ from WT and KO mice using the HFS-LTD protocol ( $\mathbf{D}$ and $\mathbf{H})$ and the DHPG-LTD protocol (L and $\mathbf{P})$ (last 10 minutes PPR relative to baseline). D, D1 MSNs: WT and KO, $P=0.15 ; \mathbf{H}, \mathrm{D} 2 \mathrm{MSNs}$ : WT and KO, $P=0.0002 ; \mathbf{L}, \mathrm{D} 1 \mathrm{MSNs}$ : WT and KO, $P=0.36 ; \mathbf{P}, \mathrm{D} 2 \mathrm{MSNs}$ : WT and KO, $P=0.0072$. An unpaired $t$ test was used for comparisons. ${ }^{*} P<0.05$; ${ }^{* *} P<0.01$; ${ }^{* * *} P<0.001$. $n$, cell number; $N$, animal number.

novaleric acid (DL-APV) $(50 \mu \mathrm{M})$ and $\mathrm{GABA}_{\mathrm{A}}$ receptor antagonist PTX $(100 \mu \mathrm{M})$. However, D2 MSNs from Shank3B-KO mice showed a significantly reduced magnitude of DHPG-induced LTD (Figure 2, M-P). In contrast, DHPG application induced comparable LTD in D1 MSNs of WT and Shank3B-KO mice (Figure 2, I-L).

MSNs coexpress 2 subclasses of L-type calcium channels, referred to as Cav1.2 and Cav1.3, that are characterized by their distinct pore-forming $\alpha$ subunits $\alpha 1 \mathrm{C}$ and $\alpha 1 \mathrm{D}$, respectively (66-68). Previous studies by Olson et al. (69) demonstrated that the 2 channels differ in their affinity for dihyhropyridine (DHP) antagonists in acutely isolated striatal MSNs. In particular, Olson et al. found that a low concentration of nimodipine $(1 \mu \mathrm{M})$ produces a near complete block of Cav1.2 channels in MSNs from Cav1.3-knockout mice and that Cav1.3 channel-mediated currents could be operationally defined as those blocked by $10 \mu \mathrm{M}$ nimodipine, but not 1 $\mu \mathrm{M}$ nimodipine. We applied the same method to determine whether Shank3 deletion could affect Cav1.3 L-type calcium channel activity in Shank3B-KO MSNs (Figure 3A). We found that Cav1.3 channel currents elicited in D2 MSNs of Shank3B-KO mice were significantly reduced compared with those of WT mice, but this is not the case for Cav1.2 channel-mediated currents (Figure 3, D and E). In contrast, in D1 MSNs, neither Cav1.2 nor Cav1.3 channel currents showed significant differences between WT and Shank3B-KO mice (Figure 3, B and C). Thus, the current density of Cav1.3 channels in D2 MSNs is selectively affected in Shank3B-KO mice.

A previous study (65) showed that strong activation of L-type calcium channels by the agonist FPL64176 was sufficient to induce LTD at striatal excitatory synapses (FPL-LTD) and that this type of LTD shared a retrograde eCB signaling mechanism with HFS/ DP-LTD. As expected, application of FPL64176 (500 nM) to striatal slices induced robust LTD in WT D1 and D2 MSNs (Figure 3, F and I). Similarly, D1 MSNs in Shank3B-KO mice exhibited robust FPL-LTD (Figure 3, F-H). In contrast, the same induction protocol resulted in impaired LTD in D2 MSNs from Shank3B-KO mice. Instead of depression, a late potentiation in some D2 MSNs was observed (Figure 3, I-K), indicating an altered calcium concentration and/or dynamics due to reduced Cav1.3 activity in D2 MSNs of Shank3B-KO mice. Furthermore, the increase of PPR of eEPSCs following application of FPL was observed only in D2 MSNs from WT mice, but not in those from Shank3B-KO mice (Figure 3K). Of note, we also observed a trend toward an increase in the decay time constant $\left(\tau_{\mathrm{w}}\right)$ of the average EPSCs after FPL application in about half of the D2 MSNs of Shank3B-KO mice (cells shown as LTP instead of LTD; WT-before: $13.5 \pm 3.30 \mathrm{~ms}$, WT-after: $15.7 \pm$ $2.77 \mathrm{~ms}, n=6$ cells, $N=3$ mice. KO-before: $12.7 \pm 2.10 \mathrm{~ms}$, KOafter: $24.83 \pm 6.00 \mathrm{~ms}, n=7$ cells, $N=3$ mice; 2 -way ANOVA, $P=$ 
A

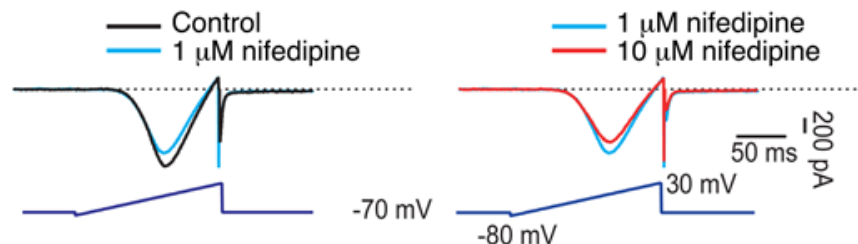

D1 MSNs

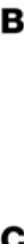

,
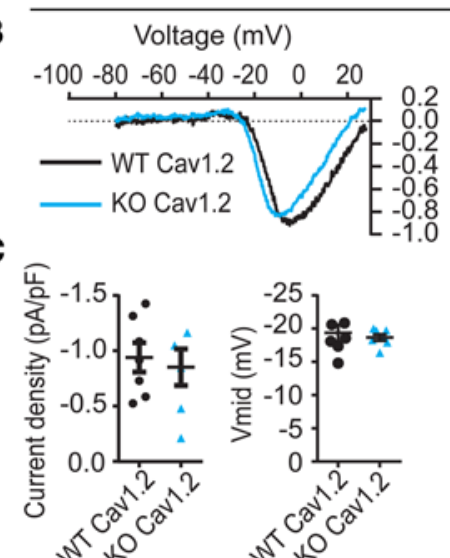
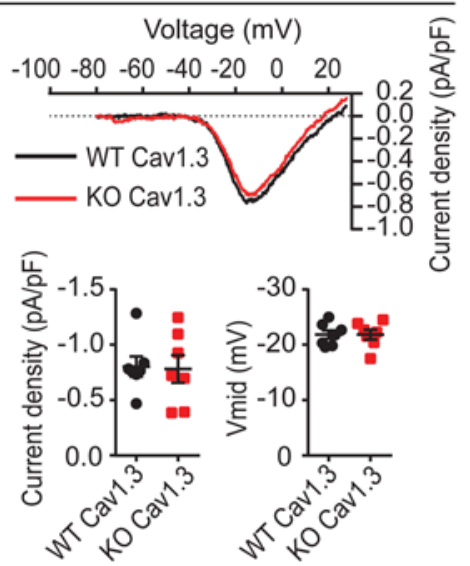

D2 MSNs

\section{D}

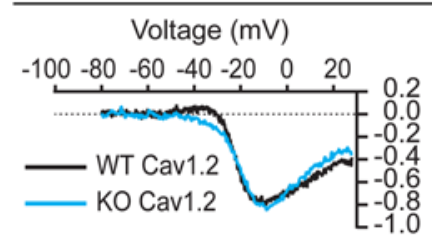

E
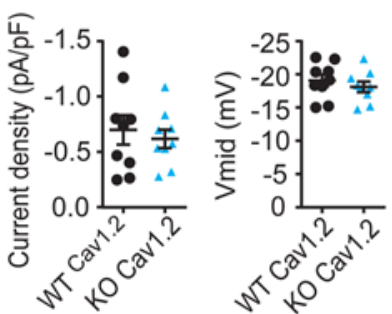
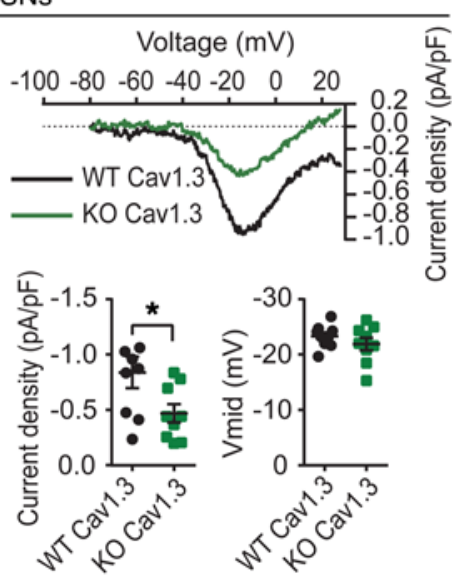

$\mathbf{F}$
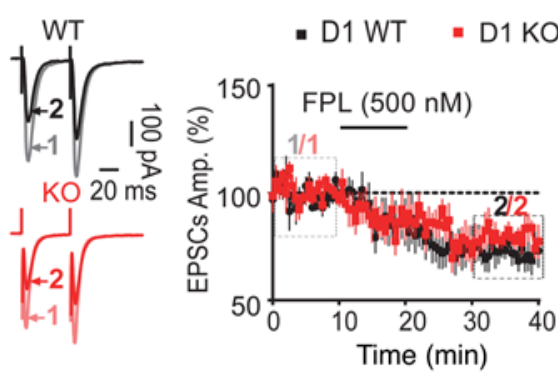

I

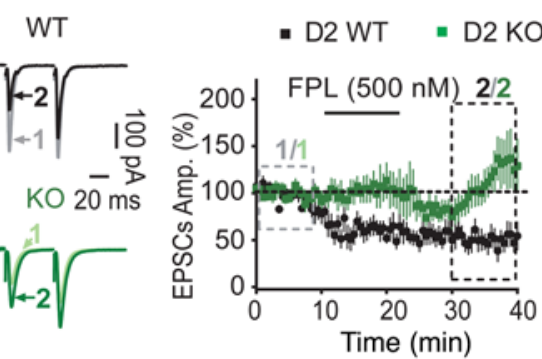

G

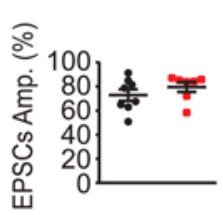

H

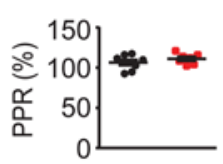

$\mathbf{J} \cong$

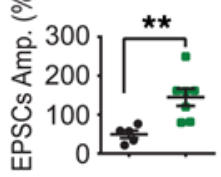

K

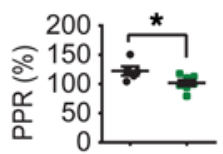

Figure 3. Shank3 deletion selectively impaired Cav1.3 function in D2 MSNs, but not D1 MSNs. (A) Protocol used to separate Cav1.2and Cav1.3-mediated current. (B and $\mathbf{D})$ Representative recordings of Cav1.2 and Cav1.3 in WT and KO D1 MSNs (B) and D2 MSNs (D). (C and $\mathbf{E}$ ) Cav1.3 channel current density was only reduced in KO D2 MSNs (C, left: D1 current density, Cav1.2: WT, $n=7, N=2 ; \mathrm{KO}, n=7$, $N=2, P=0.69$; Cav1.3: WT, $n=7, N=2 ; \mathrm{KO}, n=7, N=2, P=0.89$; C, right: D1 half-activation voltage (Vmid) Cav1.2: WT and KO, $P=0.62$; Cav1.3: WT and KO, $P=1.0$; E, left: D2 current density, Cav1.2: WT, $n=9, N=2 ; \mathrm{KO}, n=9, N=2, P=0.61 ;$ Cav1.3: WT, $n=9, N=2 ; \mathrm{KO}$, $n=9, N=2, P=0.0395 ; \mathbf{E}$, right: D2 Vmid, Cav1.2: WT and K0, $P=0.44$; Cav1.3: WT and KO, $P=0.30$ ). (F and I) Time course plots of FPL64176-LTD and representative traces of WT and Shank3B KO D1 MSNs (F) and D2 MSNs (I) after FPL64176 bath perfusion. KO D2 MSNs showed impaired FPL64176-induced LTD. (G and J) Summary data of eEPSC amplitudes (Amp.) of D1 MSNs (G) and D2 MSNs (J) from WT and KO mice (last 10 minutes eEPSC amplitude relative to baseline; C, D1 MSNs: WT, $n=8, N=6$. KO, $n=7, N=4, P=0.31$. J, D2 MSNs: WT, $n=5, N=3 ; \mathrm{KO}, n=7, N=3, P=0.0064$ ). (H and K) Summary data of PPR of D1 MSNs (H) and D2 MSNs (K) from WT and Shank3B-KO mice (last 10 minutes PPR relative to baseline; $\mathbf{H}$ D1 MSNs: WT and KO, $P=0.29$. K, D2 MSNs: WT and KO, $n=7$; $P=0.040$ ). An unpaired $t$ test was used for comparisons. ${ }^{*} P<0.05$; ${ }^{*} P<0.01$. $n$, cell number; $N$, animal number.

0.1795, Supplemental Figure 4). While the reason for the slower decay kinetic is still unclear, a possible explanation for the LTP in the subgroup of D2 MSNs of Shank3B-KO mice might be an increase in the expression of GluR2-lacking AMPARs at the synaptic sites (70-72), where a reduction of GluR2 proteins in the PSD of Shank3B-KO mice was observed in our previous study (31). Together, these data demonstrate a striatal D2 MSN-specific plasticity defect in Shank3B-KO mice.

Because of the postsynaptic localization of SHANK3 protein, the defective striatal LTD in D2 MSNs of Shank3BKO mice most likely has a postsynaptic origin, consistent with the upstream signaling pathways required for eCB generation. However, it is possible that deletion of Shank3 may directly affect presynaptic CB1R function. Thus, we specifically investigated whether signaling via presynaptic CB1Rs was altered in Shank3B-KO D2 MSNs by directly activating CB1Rs with WIN 55212-2 (1 $\mu \mathrm{M})$, a CB1R-specific agonist. We found that application of WIN-55212-2 at a concentration eliciting submaximal levels of synaptic depression resulted in a similar reduction of eEPSC amplitude in D2 MSNS of WT and Shank3B-KO mice (Supplemental Figure 5 , A and B). Consistent with its effect on presynaptic release properties, WIN 55212-2 also similarly increased the PPR ratio in WT and Shank3B-KO D2 MSNs (Supplemental Figure $5 \mathrm{C}$ ). These results suggest that there are no significant alterations in CB1R-mediated signaling itself in Shank3BKO D2 MSNs and that the changes found in striatal LTD are most likely due to the altered $\mathrm{eCB}$ signaling activity originating from the postsynaptic side.

D2 MSNs are more vulnerable to structural alterations upon Shank3 deletion. Shank3 gene mutations identified in autism have been found to affect dendritic spine development and morphology through an actin-dependent mechanism (73-76). Our previous studies found reduced spine 
A

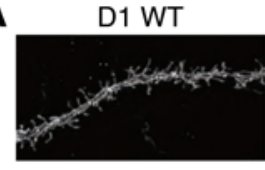

c

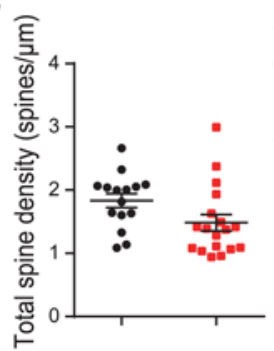

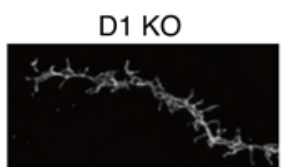

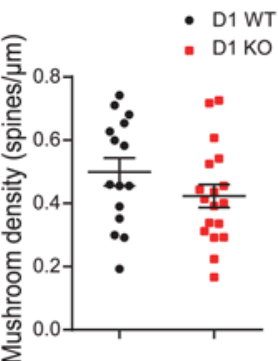

B

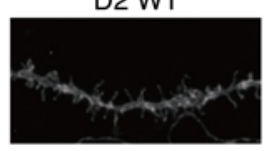

D

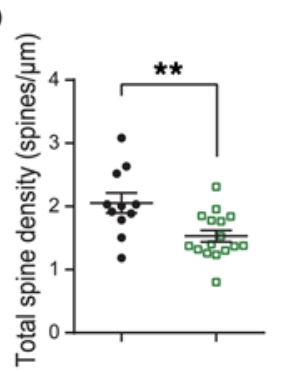

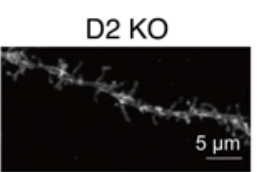

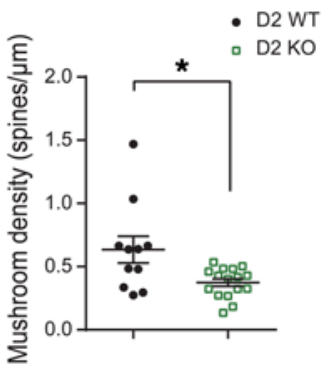

Figure 4. Reduced spine density in D2 MSNs of Shank3B-KO mice. (A and B) Representative spine images of D1 (A) and D2 (B) MSNs from WT and KO MSNs. Scale bar: $5 \mu \mathrm{m}$. (C and D) Bar graphs summarizing total spine density and mushroom-shaped spine density of WT and KO MSNs. (C) D1 MSNs, total spine density: WT and KO, $n=18, P=0.06$. Mushroom-shaped spine density: WT and KO, $n=18, P=0.19$. (D) D2 MSNs, total spine density: WT and KO, $n=16, P=0.0052$. Mushroom-shaped spine density: WT and $\mathrm{KO}, n=16, P=0.01$. An unpaired $t$ test was used for comparisons. ${ }^{*} P<0.05 ;{ }^{*} P<0.01$. $n$, dendritic segments number.

density in MSNs of Shank3B-KO mice. Here, we examined whether Shank3 deletion differentially affected spine density in D1 MSNs versus D2 MSNs. We applied a modified viral-based method to sparsely label striatal neurons using a membrane-targeted EGFP (EGFPf) delivered with an AAV viral vector (AAV-hSyn1-EGFPfWPRE-hGH), which could pass the blood-brain barrier to infect neurons when injected into the retroorbital venous sinus (77). We injected AAV-hSyn1-EGFPf-WPRE-hGH into 2- to 4-month-old Shank3B-KO/Drd1a-tdTomato and WT/Drd1a-tdTomato mice and imaged MSNs (Supplemental Figure 6). We quantified the total and mushroom-shaped spine density of both D1 (tdTomato positive) and D2 (tdTomato negative) MSNs in the dorsal striatum of WT and Shank3B-KO mice. We found that both the total and mushroom-shape spine density in D2 MSNs of Shank3B-KO mice were significantly reduced compared with those of WT mice (Figure 4, B and D), whereas only a trend of reduction was observed in D1 MSNs of Shank3B-KO mice (Figure 4, A and C). In addition, we traced striatal D1 versus D2 MSNs to investigate the cellular morphology and dendritic complexity of these cells in both WT and Shank3B-KO mice. Sholl analysis revealed a reduced dendritic complexity in D2 MSNs of Shank3B-KO mice as compared with WT mice. Again, D1 MSNs of KO mice showed levels of dendritic complexity comparable to those of WT mice (Supplemental Figure 7).

Enhancing the activity of D2 MSNs, but not D1 MSNs, rescued repetitive grooming behavior in Shank3B-KO mice. Our above results suggest that Shank3 deletion leads to more prominent synaptic defects in D2 MSNs. Since coordinated and balanced activity of the direct and indirect pathways is critical for normal striatum-mediated actions $(47,78,79)$, we set out to test whether D2 MSN dysfunction plays a causative role in repetitive grooming behavior observed in Shank3B-KO mice. To enhance the activity of D2 MSNs, we used the stimulatory designer receptors exclusively activated by designer drugs (DREADD-hM3Dq, where hM3Dq indicates Gq-coupled human M3 muscarinic receptor), which couples through a Gq pathway to depolarize neuronal membrane potential upon the application of synthetic ligand clozapine- $N$-oxide (CNO) (80-82). We bilaterally injected the Cre-dependent AAV8-hSyn-DIO-hM3DqmCherry (hM3Dq-mCherry) into the dorsal striatum of WT/Drd1aCre, Shank3B-KO/Drd1a-Cre, WT/A2a-Cre, and Shank3B-KO/A2aCre mice to selectively express hM3Dq-mCherry in D1 or D2 MSNs. As shown in Figure 5A, mCherry was specifically detected in dorsal striatal MSNs with no expression in other brain regions. In parallel, all control mice shown in Figure 5 had received bilateral injections of AAV8-hSyn-DIO-mCherry targeting the dorsal striatum. To test the functionality of DREADD-hM3Dq in MSNs, we performed whole-cell current clamp recordings of hM3Dq-mCherry-positive MSNs in acute brain slices. We found that hM3Dq-mCherryexpressing MSNs, but not control hSyn-mCherry-expressing cells, exhibited an increased excitability upon local application of $\mathrm{CNO}$, as evidenced by a marked membrane depolarization and increased firing rate in hM3Dq-mCherry-positive MSNs (Figure 5B).

We next examined whether enhancing the activity of D2 MSNs by DREADD-hM3Dq could rescue repetitive grooming behavior in Shank3B-KO mice. Because strong activation of D2 MSNs can lead to motor inhibition, as demonstrated by the optogenetic activation study (83), and thus confound our behavioral analysis, we tested the effect of different doses of CNO on motor activity in WT/A2a-Cre and Shank3B-KO/A2a-Cre mice injected with AAV8-hSyn-DIO-hM3Dq-mCherry. We found that administration of CNO at concentrations of $0.7 \mathrm{mg} / \mathrm{kg}$ or $1.0 \mathrm{mg} / \mathrm{kg}$, commonly used doses in mice, caused significant inhibition of motor activity (Supplemental Figure 8). Conversely, administration of CNO at a concentration of $0.7 \mathrm{mg} / \mathrm{kg}$ or $1.0 \mathrm{mg} / \mathrm{kg}$ (data not shown) to WT/Drd1a-Cre and Shank3B-KO/Drd1a-Cre mice injected with AAV8-hSyn-DIO-hM3Dq-mCherry dramatically increased motor activity (Supplemental Figure 8). We thus used a lower dose of CNO ( $0.3 \mathrm{mg} / \mathrm{kg}$ body weight) for our behavioral experiments, aiming to correct the reduced activity of D2 MSNs in Shank3B-KO mice without inducing significant motor activity changes. We found that administration of $\mathrm{CNO}$ at a concentration of $0.3 \mathrm{mg} / \mathrm{kg}$ to Shank3B-KO/A2a-Cre mice injected with AAV8hSyn-DIO-hM3Dq-mCherry significantly reduced self-grooming 
A

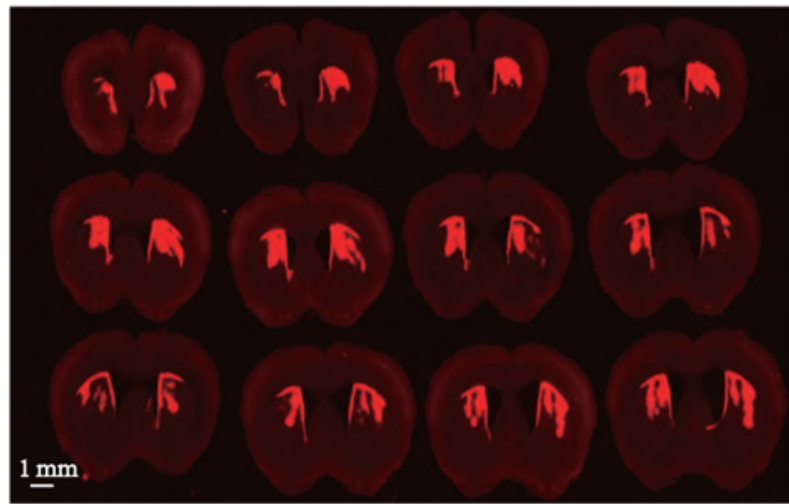

\begin{tabular}{|c|c|c|c|}
\hline$\longleftarrow 1$ Hour $\rightarrow$ & \multicolumn{3}{|c|}{$0.3 \mathrm{mg} / \mathrm{kg}$ CNO } \\
\hline Habituation & Pre-CNO & Break & Post-CNO \\
\hline & $\begin{array}{c}\text { Pre } \\
\text { Grooming \& } \\
\text { Open field test }\end{array}$ & & $\begin{array}{c}\text { Post } \\
\text { Grooming \& } \\
\text { Open field test }\end{array}$ \\
\hline
\end{tabular}

D1 MSNs
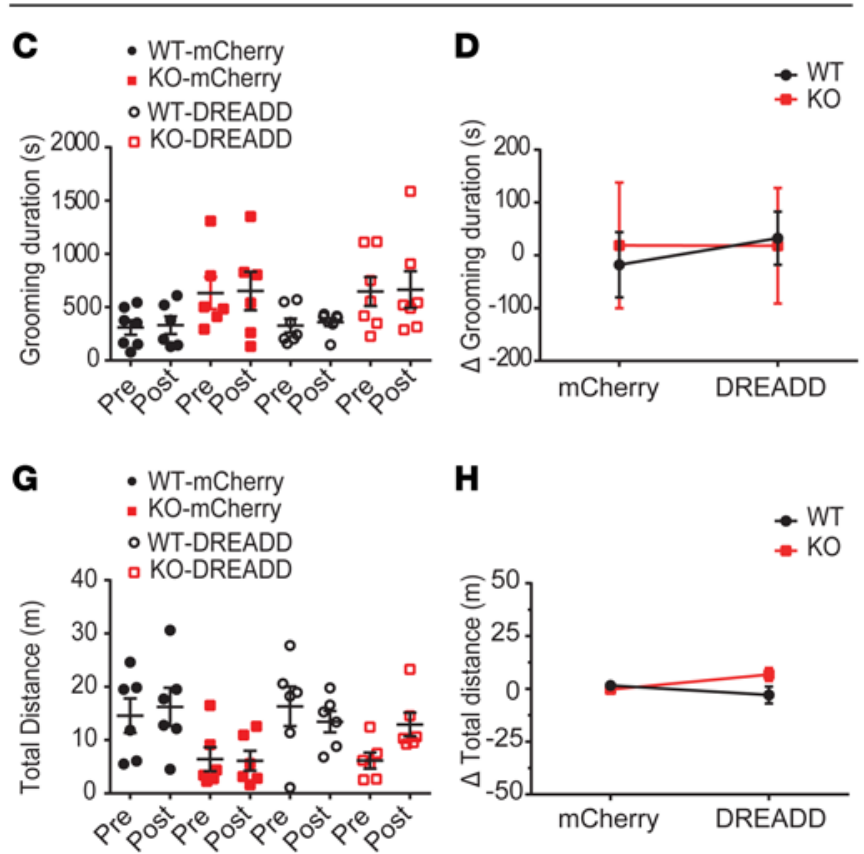

B

D1-Cre AAV8-hSyn-DIO-hM3D(Gq)

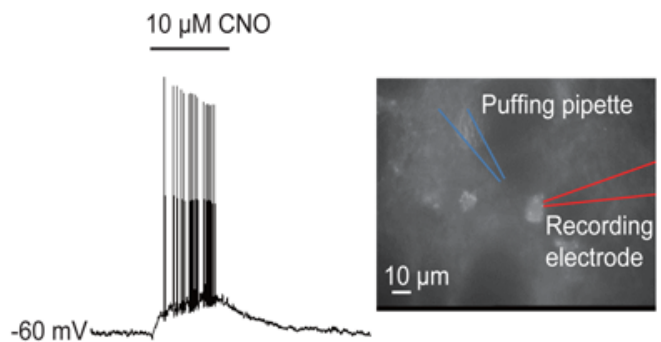

A2a-Cre AAV8-hSyn-DIO-hM3D(Gq)

$10 \mu \mathrm{M}$ CNO

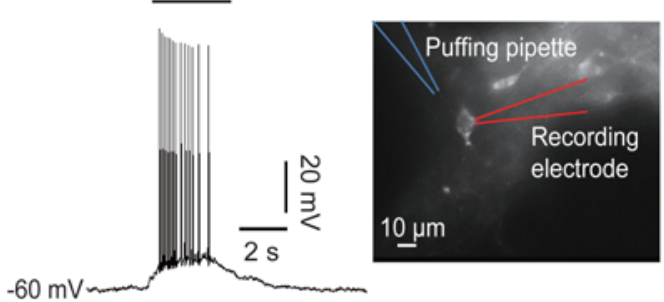

D2 MSNs
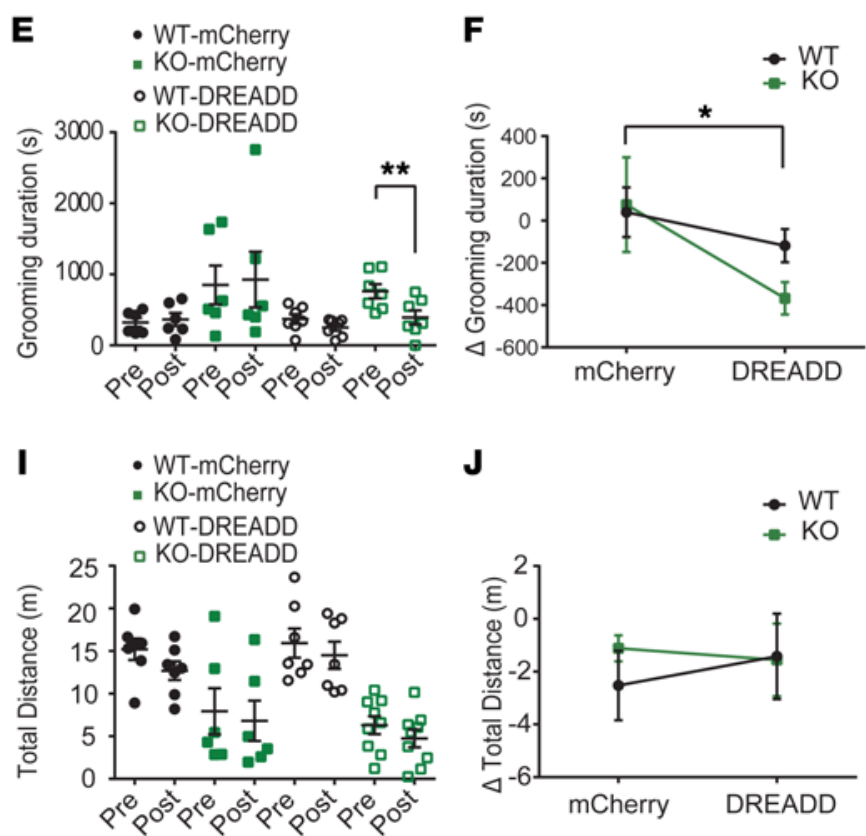

Figure 5. Enhancing D2 MSN activity using DREADD-hM3Dq reduced repetitive grooming behavior in Shank3B-KO mice. (A) The injecting site of AAVDIO-hM3Dq-mCherry virus and behavior paradigm. (B) Representative traces of CNO-induced neuronal membrane depolarization of a hM3Dq-mCherryexpressing D1 (upper panel) or D2 MSN (lower panel). (C and $\mathbf{E}) \mathrm{A}$ reduction in grooming time between pre- and post-CNO treatment only was found in KO/ A2a-Cre mice. (C, D1: WT-mCherry, $N=7, P=0.78$; KO-mCherry, $N=6, P=0.88$; WT-hM3Dq, $N=7, P=0.54$; KO-hM3Dq, $N=7, P=0.87$; E, D2: WT-mCherry, $N=6, P=0.75$; KO-mCherry, $N=6, P=0.75 ;$ WT-hM3Dq, $N=7, P=0.18$; KO-hM3Dq, $N=7, P=0.0031$ ). (D and F) DREADD significantly decreased grooming changes in KO/A2a-Cre mice (F, WT and KO, $P=0.43$. mCherry and DREADD, $P=0.03$; interaction, $P=0.29$, 2-way ANOVA), but not D1-Cre mice (D, WT and KO, $P=0.9$. mCherry and DREADD, $P=0.78$; interaction, $P=0.78$ ). (G and I) Summary data showing no changes in locomotor activity between pre- and postCNO treatment in WT/D1-Cre or KO/D1-Cre mice (G, WT-mCherry, $N=6, P=0.33$; KO-mCherry, $N=6, P=0.89$; WT-hM3Dq, $N=6, P=0.18$; KO-hM3Dq, $N=6$, $P=0.08$.) and WT/A2a-Cre or KO/A2a-Cre mice (I, WT-mCherry, $N=7, P=0.10 ;$ KO-mCherry, $N=6, P=0.07 ;$ WT-hM3Dq, $N=7, P=0.41 ; \mathrm{KO}$-hM3Dq, $N=9$, $P=0.29$ ). (H and J) DREADD did not change locomotor activities in both D1-Cre mice (H, WT and KO, $P=0.18$. mCherry and DREADD, $P=0.66$; interaction, $P=0.05$ ) and A2a-Cre mice (J, WT and KO, $P=0.64$. mCherry and DREADD, $P=0.81$; interaction, $P=0.57)$. A paired $t$ test was used for $\mathbf{C}, \mathbf{E}, \mathbf{G}$, and $\mathbf{I}$ and 2-way ANOVA was used for $\mathbf{D}, \mathbf{F}, \mathbf{H}$, and $\mathbf{J}$. ${ }^{*} P<0.05$; ${ }^{* *} P<0.01$. $N$, animal number. 
(Figure 5, E and F) without significantly affecting motor activity (Figure 5, I and J). On the other hand, enhancing the activity of D1 MSNs by CNO in Shank3B-KO/Drd1a-Cre mice injected with AAV8-hSyn-DIO-hM3Dq-mCherry did not affect self-grooming behavior (Figure 5, C and D); instead, the mice showed a trend of increased motor activity (Figure 5, G and H). Thus, enhancing D2 MSN activity, but not D1 MSN activity, is sufficient to correct the repetitive grooming behavior in Shank3B-KO mice.

Finally, we tested whether reducing D2 MSN activity in WT mice may increase repetitive grooming. To reduce the activity of D2 MSNs, we used the inhibitory DREADD (DREADD-hM4Di), which couples through a Gi pathway to hyperpolarize neuronal membrane potential upon the application of CNO. We bilaterally injected Cre-dependent AAV8-hSyn-DIO-hM4Di-mCherry (hM4Di-mCherry) into the dorsal striatum of WT/A2a-Cre mice to selectively express hM4Di-mCherry in D2 MSNs. To test the functionality of DREADD-hM4Di in MSNs, we performed wholecell current clamp recordings of hM4Di-mCherry-positive MSNs in acute brain slices. We found that hM4Di-mCherry-expressing MSNs exhibited a hyperpolarization upon local application of CNO (Supplemental Figure 9, A and B). We then tested the effect of CNO $(0.3 \mathrm{mg} / \mathrm{kg})$ on repetitive grooming behavior of WT/A2aCre mice bilaterally injected with hM4Di-mCherry. In parallel, all control WT/A2a-Cre mice (without hM4Di-mCherry viral injection) were treated with the same dosage of $\mathrm{CNO}$ and tested for grooming duration. As shown in Supplemental Figure 9C, no significant changes in grooming duration were observed between the 2 groups. Although these results suggest that a moderate reduction in D2 MSN activity alone could not induce repetitive grooming in WT mice, other explanations also exist. First, rescuing behavioral defects may only require the normalization of a subset of neurons in the circuit, while generating behavioral defects may require a larger subset of abnormally functioning neurons. Second, there might be some compensatory mechanisms that could regain a balanced activity of the 2 striatal pathways in WT mice, whereas in Shank3B-KO mice, such compensatory mechanisms might be compromised and imbalanced activity of the 2 striatal pathways could eventually lead to the repetitive grooming behavior.

\section{Discussion}

In the present work, we found synaptic defects in both D1 MSNs and D2 MSNs in Shank3B-KO mice. However, Shank3 deletion seems to preferentially affect synapses in D2 MSNs, including changes in presynaptic and postsynaptic function as well as spine density. In D1 MSNs, Shank3 deletion only reduces postsynaptic AMPAR response. To the best of our knowledge, this is the first study to provide direct evidence that a primary dysfunction in the striatal indirect pathway could give rise to repetitive behavior in a mouse model of autism. Previous studies have suggested that an imbalance of the direct and indirect pathways of the basal ganglia may underlie repetitive behavior $(78,79,84)$. Recently, it was shown in neuroligin-1 and neuroligin-3 mutant mice that abnormality of D1 MSNs can lead to autistic-like behaviors, including repetitive behavior $(85,86)$. Our present study in Shank3B mutant mice demonstrates that hypoactivity of D2 MSNs is also sufficient. It will be interesting to determine whether modulating D2 MSN activity could also correct repeti- tive behaviors other than self-grooming. However, the high levels of anxiety and locomotion defects in Shank3B mutant mice complicate other repetitive behavior tests such as marble burying (data not shown). Future studies with other ASD models will be needed to address this question.

Currently, the molecular mechanisms of how Shank3 loss causes the differential structural and functional alterations between D1 MSNs and D2 MSNs are unknown. Although Shank3 is expressed in both D1 MSNs and D2 MSNs (69), D1 MSNs and D2 MSNs have differential transcriptome profiles, including differential expression of many signaling proteins $(87,88)$. Therefore, since SHANK3 is a major scaffolding protein, one possible explanation is that SHANK3-containing scaffolds form distinct signaling complexes in D1 MSNs and D2 MSNs and that disruption of these complexes may lead to distinct synaptic defects. Alternatively, the Shank3 gene has multiple intragenic promoters and extensive alternative splicing, thereby producing multiple isoforms in the mouse brain $(33,75,89)$. These different isoforms may be differentially expressed in D1 MSNs and D2 MSNs. Based on the presence of unique protein-binding domains in each isoform, distinct signaling complexes could form and thus result in distinct defects in the 2 types of MSNs. Recent work from Wang et al. (75) provides direct evidence demonstrating that Shank3 isoforms display different subcellular localization and differential effects on dendritic and spine morphology, further supporting this hypothesis. Thus, it is tempting to speculate that deletion of Shank3 leads to the disruption of distinct synaptic signaling complexes in D1 and D2 MSNs due to differential binding partners in these 2 types of cells. The disruption of SHANK3 signaling in D2 MSN affects both pre- and postsynaptic function, while Shank3 deletion in D1 MSNs only causes a postsynaptic change of reduced synaptic AMPAR response. In addition, reduced neuronal dendritic complexity is only found in D2 MSNs. The reduced dendritic complexity result appeared to be inconsistent with our previous report, where a hypertrophy of striatal neuronal morphology was found in Shank3 mutant mice (31). While we don't know the exact reasons for the seemingly inconsistent dendritic complexity results, our previous study was done on 5-week-old mice, whereas 3- to 5-monthold mice were used in the current study. In addition, in our prior study, we collected a mixed population of both D1 and D2 MSNs. Considering the intrinsically different dendritic structures with higher dendritic complexity in D1 MSNs than in D2 MSNs (shown in Supplemental Figure 7), a confounding effect from the MSN cell types other than genotype might also contribute to the discrepancy. It will be important to directly test the functional roles of Shank3 isoforms at specific synapses and in different cell types during development and to assess the relevance of the difference isoforms to the neural circuit dysfunction that underlies autisticlike behaviors.

Defects of mGluR5 signaling have been found in several mouse models of ASD (90-92). SHANK3 is known to interact with mGluR5 via the Homer adaptor protein. Given the fact that eCB generation requires the activation of postsynaptic calcium signaling and mGluR5, it is not surprising that we found defects in eCBmediated long-term synaptic plasticity in D2 MSNs of Shank3BKO mice. While both Shank3B-KO mice and the $T s c 2^{+/-}$mouse model of tuberous sclerosis complex exhibited a reduction in 
mGluR-induced LTD, the fragile X syndrome (FXS) mouse model (Fmr1-KO mice) showed excessive group I mGluR activation, leading to an increased mGluR-induced LTD $(90,93)$. Although it is not clear whether and how the observed changes in striatal LTD in Shank3B-KO mice are linked to autistic-like behaviors such as repetitive grooming and social interaction deficits, our results further support the notion that mGluR5 signaling abnormalities might be a common phenomenon in a subset of ASD models (92). In addition, the loss of eCB-LTD could be due to abnormal eCB signaling in the Shank3B mutant mice that occludes induction of further LTD. It is well established that eCB-LTD can be developmentally regulated. Considering the very recent work that demonstrated an early hyperactivity of corticostriatal circuits in Shank3B$\mathrm{KO}$ mice (94), it is possible that a type of abnormal eCB signaling has formed during development. It thus will be interesting for our future studies to explore the potential abnormal eCB signaling in development in Shank3B-KO mice.

One prominent hypothesis posits that repetitive behavior is a consequence of relative hyperactivity along cortico-basal ganglia-cortical feedback circuits, implicating an imbalanced activity between the 2 striatal pathways in the basal ganglia, which eventually facilitate the release of compulsive motor programs $(84,95$, 96). Our findings, in particular the correction of repetitive grooming by enhancing D2 MSN activity with the DREADD system in an attempt to regain balanced activity between the 2 striatal pathways in Shank3B-KO mice, largely support such an activity-imbalance theory. However, it is worth noting that the moderate enhancement of D1 MSN activity with the DREADD system by applying low-dose CNO $(0.3 \mathrm{mg} / \mathrm{kg})$ did not induce or exacerbate repetitive grooming behavior in either WT or Shank3B-KO mice (Figure 5, C and D). Instead, it only induced a trend of motor activity enhancement in Shank3B-KO mice, but not in WT mice (Figure 5, G and H). One possible explanation is that Shank3B-KO mice might be more sensitive to such moderate manipulation than WT mice due to their disrupted dual-pathway balanced activity toward a relative higher activity of direct pathway. Interestingly, such moderate enhancement of indirect pathway activity did not lead to differential changes in motor activity between WT and Shank3B-KO mice (Figure 5, I and J). Instead, we only observed the rescue of repetitive grooming behavior in Shank3B-KO mice (Figure 5, E and F). Thus, our results suggest a much more complex scenario than the simple activity imbalance hypothesis. It is possible that, besides the importance of balanced and cooperative activity of the 2 striatal pathways, indirect pathway activity might be more engaged in the mediation of repetitive behavior, while changes in direct pathway activity might have a stronger effect on locomotor activity. Indeed, as shown by optogenetic manipulations (83) of MSN activities, bilateral activation of direct-pathway MSNs elicited an increase in ambulation and reduction of fine movements, but changes in such movements were not correlated with changes in grooming frequency. Such pathwayspecific engaging function could also explain the distinct rescue of locomotion defects when D1 MSN activity, but not D2 MSN activity, is enhanced in Shank3B-KO mice (Supplemental Figure 8, C and E) when they are given high doses of CNO $(0.7 \mathrm{mg} / \mathrm{kg})$.

Previous pharmacological studies and genetic modulation of dopaminergic genes (such as the dopamine transporter DAT and the $\mathrm{D} 1$ receptor) indicated that enhanced activity of the direct path- way induced repetitive behavior (97-100). However, a potential complication for such interpretation is the widespread expression of dopamine receptors outside the striatum, such as in the cortex and limbic regions (96). Unlike the complexity of pharmacological and genetic modulation through dopaminergic receptors, the striatal cell-type-specific activation of direct pathway D1 MSNs either by optogenetic or the chemical-genetic DREADD approach did not appear to induce repetitive or stereotyped behavior $(82,83,101)$, which is in agreement with earlier works suggesting that activating the direct pathway leads to hyperactivity, not stereotypy $(102,103)$. Overall, our studies highlight the importance of dissecting the unique functional role of each pathway individually as well as the interplay between the two in order to understand the mechanisms underlying repetitive behaviors seen in many psychiatric disorders.

\section{Methods}

Animals. The generation of Shank3B-KO mice was described previously (31). Experimental mice were the progeny of Shank3B $\mathrm{B}^{+-} /$Drd1atdTomato or Drd2-EGFP breeding pairs (with C57BL/6 genetic background, backcrossing for at least 10 generations) in which only 1 of the parents expressed the Drd1a-tdTomato or Drd2-EGFP transgenes, so that no experimental subjects were homozygous for the transgenic fluorescent markers $(51,52)$. In experiments testing genotype effects, littermate control animals of both sexes were used (age around 1.5 to 2 months). All experiments were performed and analyzed with the experimenter blinded to genotypes.

Electrophysiological studies. Six- to eight-week-old mice of both sexes were used for all whole-cell electrophysiology procedures. Acute coronal brain slices were prepared as follows. Mice were anesthetized with Avertin solution $(20 \mathrm{mg} / \mathrm{ml}, 0.5 \mathrm{mg} / \mathrm{g}$ body weight) and transcardially perfused with 15 to $20 \mathrm{ml}$ of ice-cold carbogenated $\left(95 \% \mathrm{O}_{2}, 5 \% \mathrm{CO}_{2}\right)$ cutting solution containing the following: $194 \mathrm{mM}$ sucrose, $30 \mathrm{mM} \mathrm{NaCl}, 4.5 \mathrm{mM} \mathrm{KCl}, 1.2 \mathrm{mM} \mathrm{NaH}_{2} \mathrm{PO}_{4}, 0.2$ $\mathrm{mM} \mathrm{CaCl}_{2}, 2 \mathrm{mM} \mathrm{MgCl}_{2}, 26 \mathrm{mM} \mathrm{NaHCO}_{3}$, and $10 \mathrm{mM} \mathrm{D}$-(+)-glucose (with osmolarity of 340-350 mOsm). The brains were then rapidly removed and placed in ice-cold cutting solution for slice preparation. Coronal slices $(300 \mu \mathrm{m})$ were prepared and then incubated at $32^{\circ} \mathrm{C}$ with carbogenated artificial cerebral spinal fluid (aCSF) for 10 to 15 minutes. The slices were then incubated at room temperature for at least 1 hour in aCSF that contained the following: $119 \mathrm{mM} \mathrm{NaCl}$, $2.3 \mathrm{mM} \mathrm{KCl}, 1.0 \mathrm{mM} \mathrm{NaH}_{2} \mathrm{PO}_{4}, 26 \mathrm{mM} \mathrm{NaHCO}_{3}, 11 \mathrm{mM}$ glucose, 1.3 $\mathrm{mM} \mathrm{MgSO}_{4}$, and $2.5 \mathrm{mM} \mathrm{CaCl}_{2}$ (pH 7.4, with osmolarity of 295-305 $\mathrm{mOsm}$ ) at room temperature for at least 1 hour.

The slices were constantly perfused with carbogenated aCSF at room temperature $\left(22-24^{\circ} \mathrm{C}\right)$ for all the spontaneous synaptic activity measurements to gain better stability of the patch membrane. For all the plasticity recordings (including HFS induction protocol, DHPG, and FPL chemical induction protocol), the slices were perfused with carbogenated aCSF at $32^{\circ} \mathrm{C}$. The perfusion rate was around $3.0 \mathrm{ml} / \mathrm{min}$. The individual fluorescently positive D1 or D2 MSNs were identified with a microscope equipped with GFP or RFP filter (BX-51WI, Olympus). Whole-cell patch-clamp recordings were performed with an IR-DIC visual guide. For AMPA mEPSCs and evoked AMPAR-mediated EPSCs, pipettes were filled with the internal solution containing the following: $110 \mathrm{CsOH}$ (50\% wt), D-gluconic acid (49\%-53\% wt), $4 \mathrm{mM} \mathrm{NaCl}, 15 \mathrm{mM} \mathrm{KCl}, 5 \mathrm{mM}$ TEACl, 20 mM HEPES, 0.2 mM EGTA, 5 mM lidocaine $N$-ethyl chloride, 
$4 \mathrm{mM}$ ATP magnesium salt, and $0.3 \mathrm{mM}$ GTP sodium salt. $\mathrm{pH}$ was adjusted to 7.2-7.4 with $\mathrm{CsOH}$, and osmolarity was adjusted to 298$300 \mathrm{mOsm}$ with $\mathrm{K}_{2} \mathrm{SO}_{4}$ (approximately 8-10 mM). For $\mathrm{GABA}_{\mathrm{A}}$ receptor-mediated mIPSCs, pipettes were filled with internal solution containing the following: $103 \mathrm{mM} \mathrm{CsCl}, 12 \mathrm{mM} \mathrm{CsOH}$ (50\% wt), $12 \mathrm{mM}$ methanesulfonic acid, $4 \mathrm{mM} \mathrm{NaCl}, 5 \mathrm{mM}$ TEA-Cl, $10 \mathrm{mM}$ HEPES, $0.5 \mathrm{mM}$ EGTA, $10 \mathrm{mM}$ phosphocreatine, $5 \mathrm{mM}$ lidocaine $N$-ethyl chloride, $4 \mathrm{mM}$ ATP magnesium salt, and $0.3 \mathrm{mM}$ GTP sodium salt. $\mathrm{pH}$ was adjusted to 7.2-7.4 with $\mathrm{KOH}$, and osmolarity was adjusted to 298-300 mOsm with $\mathrm{K}_{2} \mathrm{SO}_{4}$ (approximately 8-10 $\mathrm{mM}$ ). Miniature synaptic events were not recorded until 5 minutes after entering whole-cell patch-clamp recording mode to allow the dialysis of $\mathrm{Cs}^{+}$internal solution for a relatively complete block of the potassium channels in the striatal MSNs. Cells in which the series resistance (Rs, typically 8-12 M $\Omega$ ) changed by more than $20 \%$ were excluded for data analysis. In addition, cells with Rs of more than $20 \mathrm{M} \Omega$ at any time during the recordings were discarded. Cell membrane potential was held at $-70 \mathrm{mV}$ unless otherwise specified, with a Multiclamp 700B amplifier (Molecular Devices). Signals were low-pass filtered at $2 \mathrm{kHz}$ and sampled at $10 \mathrm{kHz}$ with a Digidata 1440A (Molecular Devices), and data were stored on a computer for subsequent off-line analysis. mEPSCs and mIPSCs were detected and analyzed with MiniAnalysis (Synaptosoft).

For paired-pulse stimulation experiments and LTD experiments, AMPAR-mediated EPSCs were evoked by a local concentric bipolar stimulating electrode (CBARC75, FHC). The stimulating electrode was placed in the inner edge of the corpus callosum within the dorsolateral striatum. Recordings were made in the presence of PTX (100 $\mu \mathrm{M})$ and DL-APV $(50 \mu \mathrm{M})$ to block activation of $\mathrm{GABA}_{\mathrm{A}}$ receptors and NMDARs. The stimulus intensity was set at a level that evoked 200$300 \mathrm{pA}$ of AMPAR-mediated EPSCs for all the cells measured and delivered with an interstimulus interval of $50 \mathrm{~ms}$. Paired-pulse measurements were obtained for 15 consecutive traces, and only those traces with stable evoked first current response were used for data analysis. The PPR was calculated with the peak current response to the second pulse divided by that of the first response. Only those neurons present in the baseline drifting less than $10 \%$ over 30 minutes were used to induce LTD protocol for either HFS or chemical LTD experiments.

Morphological characterization of dendritic trees and spines. To sparsely label MSNs, $25 \mu \mathrm{l}$ pAAV-hSyn1-EGFPf-WPRE-hGH virus (Penn Vector Core, University of Pennsylvania, Philadelphia, Pennsylvania, USA) with the titer of $2 \times 10^{12}(\mathrm{GC} / \mathrm{ml})$ in PBS was delivered into mouse blood circulation through retroorbital injection of the venous sinus (104). Shank3B-KO/Drd1a-tdTomato mice and WT littermates were injected with virus at the age of 2 to 4 months and euthanized 3 weeks later for immunostaining. PFA-fixed 200- $\mu$ m-thick coronal brain slices were prepared using Vibratome. Immunostaining was performed with an anti-GFP antibody (Invitrogen, catalog A11122) to visualize EGFPf expression. After staining, each slice was surrounded by a $240-\mu \mathrm{m}$ depth spacer (Electron Microscopy Sciences) and mounted on slides with nonhardening Vectashield mounting medium (Vector Laboratories, catalog H-1000).

Olympus Fluoview FV1000 confocal microscope was used to image EGFP-labeled dorsal striatal MSN cells. To acquire relatively intact dendritic trees, neurons located around the center in the depth of sections were imaged under a $40 \times$ oil lens at $1-\mu \mathrm{m}$ intervals for a total thickness of approximately $100 \mu \mathrm{m}$. Each neuron was manually traced by using Neurolucida software (MBF Bioscience) for 3D reconstruction and measurements. Stack images $(1024 \times 1024$ pixels $)$ of spines were acquired using a $60 \times$ oil lens plus $6 \times$ zoom-in. Huygens Deconvolution software (Scientific Volume Imaging) was used to process the images. Spines for the imaged dendritic segments were automatically detected and grouped into mushroom and nonmushroom using NeuronStudio software (CNIC) with a post-hoc manual correction. Experimenters were blinded to genotypes of mice during viral injection, imaging, and measurement analyses.

Viral injections for DREADD- $h M 3 D q$ rescue experiments. Each mouse was deeply anesthetized (1\%-2\% isoflurane) and was administered a dose of $1 \mathrm{mg} / \mathrm{kg}$ of meloxicam subcutaneously by injection prior to any incisions being made. The mouse was then mounted in a stereotaxic frame with nonpuncturing ear bars. AAV8-hSyn-DIO$\mathrm{hM}_{3}$ Dq-mCherry or control AAV8-hSyn-DIO-mCherry (University of North Carolina Viral Vector Core, Chapel Hill, North Carolina, USA; approximate titer $2 \times 10^{12} \mathrm{GC} / \mathrm{ml}$ ) solution was injected using a nanoinjector with injection micropipette at the following coordinates for each mouse: anterior/posterior: $1.18 \mathrm{~mm}$, medial/lateral: \pm 1.25 $\mathrm{mm}$, dorsal/ventral: $2.2 \mathrm{~mm}$ and $2.8 \mathrm{~mm}$; anterior/posterior: $1.18 \mathrm{~mm}$, medial/lateral: $\pm 1.8 \mathrm{~mm}$, dorsal/ventral: $2.2 \mathrm{~mm}$ and $3.0 \mathrm{~mm}$; anterior/posterior: $0.35 \mathrm{~mm}$, medial/lateral: $\pm 1.5 \mathrm{~mm}$, dorsal/ventral: 2.4 $\mathrm{mm}$ and $3.0 \mathrm{~mm}$; anterior/posterior: $0.35 \mathrm{~mm}$, medial/lateral: \pm 2.2 $\mathrm{mm}$, dorsal/ventral: $2.4 \mathrm{~mm}, 3.0 \mathrm{~mm}$ and $3.5 \mathrm{~mm}$. A total volume of $69 \mathrm{nl}$ was injected at each desired depth at the speed of $23 \mathrm{nl}$ per second. Incisions in the scalp were closed after injection, and animals were allowed to recover in accordance with MIT Committee on Animal Care guidelines. After surgery, mice were individually housed for 2 weeks before behavioral tests.

Behavioral experiments. Grooming behavior was measured as previously described (31). Briefly, mouse grooming was videotaped and coded from 19:00 to 21:00 hours beginning at the initiation of the dark cycle; this segment was analyzed using Noldus Observer software, and the total amount of time in the 2-hour segment spent grooming was determined. Grooming included all sequences of face wiping, scratching/rubbing of head and ears, and full-body grooming. Locomotion activity was measured using LABORAS (Metris), which relies on vibration and force signal analysis to determine both the behavior and the position of the animal (105). Three hours of continuous recordings were classified with LABORAS software v2.5 to establish locomotion activity. To test the effect of CNO on locomotion activity and grooming behavior, mice were placed in the chamber of LABORAS for 1 hour for habituation and 1 hour recording for basal behavior. Then the mice were injected with a dose of CNO and returned to the LABORAS chamber for another 2 hours of recording. The observer was blinded to genotypes during the scoring of the videotapes.

Drugs. TTX, PTX, NBQX, and DHPG were purchased from Tocris. DL-APV, WIN55,212-2 mesylate salt, and FPL64176 were purchased from Sigma-Aldrich. All drugs were made as concentrated stock solutions and diluted in aCSF to their final concentration on the experiment day and delivered with constant perfusion to the recording chamber. For drug stocks prepared with DMSO, the final DMSO concentration was less than $0.1 \%$.

CNO was purchased from Sigma-Aldrich. For experiments in mice, CNO was first dissolved in DMSO and then diluted to a final concentration with saline. The final concentration of DMSO was 
$0.5 \%$. Unless otherwise noted, the dose of CNO was $0.3 \mathrm{mg} / \mathrm{kg}$. CNO was injected i.p. at a volume of $100 \mu \mathrm{l} / 10 \mathrm{~g}$ body weight. For patchclamp recording, CNO was dissolved in DMSO at $10 \mathrm{mM}$ and then diluted into aCSF to a final working concentration of $10 \mu \mathrm{M}$.

Statistics. All data were transferred to GraphPad Prism for analysis and graphing. Electrophysiological data are presented as mean \pm SEM, and sample size refers to the number of cells analyzed unless noted otherwise. All error bars indicate SEM. Group results were compared by using a 2-tailed unpaired Student's $t$ test. Unless otherwise noted, behavioral testing data were analyzed with GraphPad Prism 6 using a 2-tailed paired $t$ test and 2-way repeated-measures ANOVA analysis, followed by Tukey's multiple comparisons test. A $P$ value of less than 0.05 was considered significant.

Study approval. All animal handling and experimental procedures were reviewed and approved by the MIT Committee on Animal Care and the Institutional Biosafety Committee of MIT as well as the Institutional Animal Care and Use Committee at the Fourth Military Medical University.

\section{Author contributions}

ZF and GF designed the study and oversaw the project. WW, C $\mathrm{Li}$, and QC performed most of the electrophysiological studies, behavioral experiments, and data analysis in the current manuscript. MSVDG performed MSN intrinsic membrane property characterization, imaged AAV sparsely labeled MSNs, and performed Sholl analysis. AYY analyzed spine density. XG, JH, KL, $\mathrm{YZ}$, and DW conducted behavioral tests and repetitive grooming behavior analysis. BG performed part of the electrophysiological studies. QZ helped design and run pilot behavioral experiments. $\mathrm{C}$ Lu did biochemical analysis. SW revised the manuscript for important intellectual content. CRG provided Drd1a-Cre mice. ZF wrote the manuscript with input from WW, C Li, and GF.

\section{Acknowledgments}

We thank Morgan Fleishman and Triana Dalia for help with animal care. We thank Alexandra Krol for critical reading of the manuscript. We thank Yehong Wan for giving statistics suggestions. WW is supported by a postdoc fellowship from the Simons Center for the Social Brain at MIT and the Natural Science Foundation of China (grant no. 81371498). SW is supported by the International Science and Technology Cooperation Program of China (grant no. 2011DFA32560. GF is supported by the NIMH (5R01MH097104), the Poitras Center for Affective Disorders Research at MIT, the Stanley Center for Psychiatric Research at the Broad Institute of MIT and Harvard, the Nancy Lurie Marks Family Foundation, the Simons Foundation Autism Research Initiative (SFARI), and the Simons Center for the Social Brain at MIT. ZF is supported by a National Alliance for Research on Schizophrenia and Depression (NARSAD) Young Investigator Grant from the Brain and Behavior Research Foundation and the Stanley Center for Psychiatric Research at the Broad Institute of MIT and Harvard.

Address correspondence to: Zhanyan Fu, Stanley Center for Psychiatric Research, Broad Institute of MIT and Harvard, 75 Ames Street, Cambridge, Massachusetts 02142, USA. Phone: 617.714.7401; E-mail: zfu@broadinstitute.org. Or to: Guoping Feng, McGovern Institute for Brain Research, Department of Brain and Cognitive Sciences, Massachusetts Institute of Technology, 77 Massachusetts Avenue, Cambridge, Massachusetts 02139, USA. Phone: 617.715.4898; E-mail: fengg@mit.edu.
1. Boeckers TM, et al. Proline-rich synapse-associated protein-1/cortactin binding protein 1 (ProSAP1/CortBP1) is a PDZ-domain protein highly enriched in the postsynaptic density. J Neurosci. 1999;19(15):6506-6518.

2. Boeckers TM, et al. Proline-rich synapse-associated proteins ProSAP1 and ProSAP2 interact with synaptic proteins of the SAPAP/GKAP family. Biochem Biophys Res Commun. 1999;264(1):247-252.

3. Du Y, Weed SA, Xiong WC, Marshall TD, Parsons JT. Identification of a novel cortactin SH3 domain-binding protein and its localization to growth cones of cultured neurons. Mol Cell Biol. 1998;18(10):5838-5851.

4. Naisbitt S, et al. Shank, a novel family of postsynaptic density proteins that binds to the NMDA receptor/PSD-95/GKAP complex and cortactin. Neuron. 1999;23(3):569-582.

5. Sheng M, Kim E. The Shank family of scaffold proteins. J Cell Sci. 2000;113( Pt 11):1851-1856.

6. Kim E, Sheng M. PDZ domain proteins of synapses. Nat Rev Neurosci. 2004;5(10):771-781.

7. Montgomery JM, Zamorano PL, Garner CC. MAGUKs in synapse assembly and function: an emerging view. Cell Mol Life Sci. 2004; 61(7-8):911-929.

8. McAllister AK. Dynamic aspects of CNS synapse formation. Annu Rev Neurosci. 2007;30:425-450.

9. Tu JC, et al. Coupling of mGluR/Homer and PSD-95 complexes by the Shank fam- ily of postsynaptic density proteins. Neuron. 1999;23(3):583-592.

10. Sala C, Piëch V, Wilson NR, Passafaro M, Liu G, Sheng M. Regulation of dendritic spine morphology and synaptic function by Shank and Homer. Neuron. 2001;31(1):115-130.

11. Hayashi MK, et al. The postsynaptic density proteins Homer and Shank form a polymeric network structure. Cell. 2009;137(1):159-171.

12. Hung AY, et al. Smaller dendritic spines, weaker synaptic transmission, but enhanced spatial learning in mice lacking Shank1. J Neurosci. 2008;28(7):1697-1708.

13. Roussignol G, et al. Shank expression is sufficient to induce functional dendritic spine synapses in aspiny neurons. JNeurosci. 2005;25(14):3560-3570.

14. Kreienkamp HJ. Scaffolding proteins at the postsynaptic density: shank as the architectural framework. Handb Exp Pharmacol. 2008;(186):365-380.

15. Baron MK, et al. An architectural framework that may lie at the core of the postsynaptic density. Science. 2006;311(5760):531-535.

16. Jeffries AR, et al. Molecular and phenotypic characterization of ring chromosome 22. Am J Med Genet A. 2005;137(2):139-147.

17. Soorya L, et al. Prospective investigation of autism and genotype-phenotype correlations in 22q13 deletion syndrome and SHANK3 deficiency. Mol Autism. 2013;4(1):18.

18. Wilson HL, et al. Molecular characterisation of the 22q13 deletion syndrome supports the role of haploinsufficiency of SHANK3/PROSAP2 in the major neurological symptoms. JMed Genet. 2003;40(8):575-584.

19. Bonaglia MC, et al. Disruption of the ProSAP2 gene in a $\mathrm{t}(12 ; 22)(\mathrm{q} 24.1 ; \mathrm{q} 13.3)$ is associated with the 22q13.3 deletion syndrome. Am J Hum Genet. 2001;69(2):261-268.

20. Durand CM, et al. Mutations in the gene encoding the synaptic scaffolding protein SHANK3 are associated with autism spectrum disorders. Nat Genet. 2007;39(1):25-27.

21. Phelan K, Betancur C. Clinical utility gene card for: deletion 22q13 syndrome. Eur J Hum Genet. 2011;19(4):4.

22. Bonaglia MC, et al. Identification of a recurrent breakpoint within the SHANK3 gene in the 22q13.3 deletion syndrome. JMed Genet. 2006;43(10):822-828.

23. Prasad C, et al. Genetic evaluation of pervasive developmental disorders: the terminal 22q13 deletion syndrome may represent a recognizable phenotype. Clin Genet. 2000;57(2):103-109.

24. Phelan MC, et al. 22q13 deletion syndrome. Am J Med Genet. 2001;101(2):91-99.

25. Abrahams BS, Geschwind DH. Advances in autism genetics: on the threshold of a new neurobiology. Nat Rev Genet. 2008;9(5):341-355.

26. Südhof TC. Neuroligins and neurexins link synaptic function to cognitive disease. Nature. 
2008;455(7215):903-911.

27. Bourgeron T. A synaptic trek to autism. Curr Opin Neurobiol. 2009;19(2):231-234.

28. Sanders SJ, et al. Insights into autism spectrum disorder genomic architecture and biology from 71 risk loci. Neuron. 2015;87(6):1215-1233.

29. Moessner R, et al. Contribution of SHANK3 mutations to autism spectrum disorder. $A m J$ Hum Genet. 2007;81(6):1289-1297.

30. Gauthier J, et al. Novel de novo SHANK3 mutation in autistic patients. Am JMed Genet B Neuropsychiatr Genet. 2009;150B(3):421-424.

31. Peça J, et al. Shank3 mutant mice display autisticlike behaviours and striatal dysfunction. Nature. 2011;472(7344):437-442.

32. Bozdagi $\mathrm{O}$, et al. Haploinsufficiency of the autism-associated Shank3 gene leads to deficits in synaptic function, social interaction, and social communication. Mol Autism. 2010;1(1):15.

33. Wang $X$, et al. Synaptic dysfunction and abnormal behaviors in mice lacking major isoforms of Shank3. Hum Mol Genet. 2011;20(15):3093-3108

34. Kouser M, et al. Loss of predominant Shank 3 isoforms results in hippocampus-dependent impairments in behavior and synaptic transmission. JNeurosci. 2013;33(47):18448-18468.

35. Yang M, et al. Reduced excitatory neurotransmission and mild autism-relevant phenotypes in adolescent Shank3 null mutant mice. J Neurosci. 2012;32(19):6525-6541.

36. Jaramillo TC, Speed HE, Xuan Z, Reimers JM, Liu S, Powell CM. Altered striatal synaptic function and abnormal behaviour in Shank3 exon4-9 deletion mouse model of autism. Autism Res. 2016;9(3):350-375.

37. Ahmari SE, et al. Repeated cortico-striatal stimulation generates persistent OCD-like behavior. Science. 2013;340(6137):1234-1239.

38. Pittenger C, Bloch MH, Williams K. Glutamate abnormalities in obsessive compulsive disorder: neurobiology, pathophysiology, and treatment. Pharmacol Ther. 2011;132(3):314-332.

39. Hollander E, et al. Striatal volume on magnetic resonance imaging and repetitive behaviors in autism. Biol Psychiatry. 2005;58(3):226-232.

40. Peterson BS, et al. A functional magnetic resonance imaging study of tic suppression in Tourette syndrome. Arch Gen Psychiatry. 1998;55(4):326-333.

41. Sears LL, Vest C, Mohamed S, Bailey J, Ranson BJ, Piven J. An MRI study of the basal ganglia in autism. Prog Neuropsychopharmacol Biol Psychiatry. 1999;23(4):613-624.

42. Saint-Cyr JA, Taylor AE, Nicholson K. Behavior and the basal ganglia. Adv Neurol. 1995;65:1-28.

43. Graybiel AM. Habits, rituals, and the evaluative brain. Annu Rev Neurosci. 2008;31:359-387.

44. Gerfen CR, et al. D1 and D2 dopamine receptor-regulated gene expression of striatonigral and striatopallidal neurons. Science. 1990;250(4986):1429-1432.

45. Kawaguchi Y, Wilson CJ, Emson PC. Projection subtypes of rat neostriatal matrix cells revealed by intracellular injection of biocytin. J Neurosci. 1990;10(10):3421-3438.

46. Cui G, et al. Concurrent activation of striatal direct and indirect pathways during action initiation. Nature. 2013;494(7436):238-242.
47. Tecuapetla F, Matias S, Dugue GP, Mainen ZF, Costa RM. Balanced activity in basal ganglia projection pathways is critical for contraversive movements. Nat Commun. 2014;5:4315.

48. Macpherson T, Morita M, Hikida T. Striatal direct and indirect pathways control decision-making behavior. Front Psychol. 2014;5:1301.

49. Mei Y, et al. Adult restoration of Shank3 expression rescues selective autistic-like phenotypes. Nature. 2016;530(7591):481-484.

50. Zhou Y, et al. Mice with Shank3 mutations associated with ASD and schizophrenia display both shared and distinct defects. Neuron. 2016; 89(1):147-162.

51. Shuen JA, Chen M, Gloss B, Calakos N. Drd1atdTomato BAC transgenic mice for simultaneous visualization of medium spiny neurons in the direct and indirect pathways of the basal ganglia. J Neurosci. 2008;28(11):2681-2685.

52. Gong S, et al. A gene expression atlas of the central nervous system based on bacterial artificial chromosomes. Nature. 2003;425(6961):917-925.

53. Koós T, Tepper JM. Inhibitory control of neostriatal projection neurons by GABAergic interneurons. Nat Neurosci. 1999;2(5):467-472.

54. Gittis AH, Nelson AB, Thwin MT, Palop JJ, Kreitzer AC. Distinct roles of GABAergic interneurons in the regulation of striatal output pathways. JNeurosci. 2010;30 (6):2223-2234

55. Calabresi P, Maj R, Pisani A, Mercuri NB, Bernardi G. Long-term synaptic depression in the striatum: physiological and pharmacological characterization. J Neurosci. 1992;12 (11):4224-4233.

56. Choi S, Lovinger DM. Decreased probability of neurotransmitter release underlies striatal longterm depression and postnatal development of corticostriatal synapses. Proc Natl Acad Sci U S A. 1997;94(6):2665-2670.

57. Kreitzer AC, Malenka RC. Dopamine modulation of state-dependent endocannabinoid release and long-term depression in the striatum. J Neurosci. 2005;25(45):10537-10545.

58 . Wang Z, et al. Dopaminergic control of corticostriatal long-term synaptic depression in medium spiny neurons is mediated by cholinergic interneurons. Neuron. 2006;50(3):443-452.

59. Kreitzer AC, Malenka RC. Endocannabinoidmediated rescue of striatal LTD and motor deficits in Parkinson's disease models. Nature. 2007;445(7128):643-647.

60 . Trusel M, et al. Coordinated regulation of synaptic plasticity at striatopallidal and striatonigral neurons orchestrates motor control. Cell Rep. 2015;13(7):1353-1365.

61. Akopian G, Musleh W, Smith R, Walsh JP. Functional state of corticostriatal synapses determines their expression of short- and long-term plasticity. Synapse. 2000;38(3):271-280.

62. Gerdeman GL, Ronesi J, Lovinger DM. Postsynaptic endocannabinoid release is critical to longterm depression in the striatum. Nat Neurosci. 2002;5(5):446-451.

63. Lovinger DM. Neurotransmitter roles in synaptic modulation, plasticity and learning in the dorsal striatum. Neuropharmacology. 2010;58(7):951-961.

64. Sung KW, Choi S, Lovinger DM. Activation of group I mGluRs is necessary for induction of long-term depression at striatal synapses. J Neu- rophysiol. 2001;86(5):2405-2412.

65. Adermark L, Lovinger DM. Combined activation of L-type $\mathrm{Ca} 2+$ channels and synaptic transmission is sufficient to induce striatal long-term depression. J Neurosci. 2007;27(25):6781-6787.

66. Hell JW, et al. Identification and differential subcellular localization of the neuronal class $\mathrm{C}$ and class D L-type calcium channel alpha 1 subunits. JCell Biol. 1993;123(4):949-962.

67. Lipscombe D, Helton TD, Xu W. L-type calcium channels: the low down. J Neurophysiol. 2004; 92(5):2633-2641

68. Xu W, Lipscombe D. Neuronal Ca(V)1.3alpha(1) L-type channels activate at relatively hyperpolarized membrane potentials and are incompletely inhibited by dihydropyridines. J Neurosci. 2001;21(16):5944-5951.

69. Olson PA, et al. G-protein-coupled receptor modulation of striatal CaV1.3 L-type Ca2+ channels is dependent on a Shank-binding domain. J Neurosci. 2005;25(5):1050-1062.

70. Angulo MC, Lambolez B, Audinat E, Hestrin $\mathrm{S}$, Rossier J. Subunit composition, kinetic, and permeation properties of AMPA receptors in single neocortical nonpyramidal cells. J Neurosii. 1997;17(17):6685-6696.

71. Isaac JT, Ashby MC, McBain CJ. The role of the GluR2 subunit in AMPA receptor function and synaptic plasticity. Neuron. 2007;54(6):859-871.

72. Asrar S, Zhou Z, Ren W, Jia Z. Ca (2+) permeable AMPA receptor induced long-term potentiation requires PI3/MAP kinases but not $\mathrm{Ca} / \mathrm{CaM}$-dependent kinase II. PLOS ONE. 2009;4 (2):e4339.

73. Durand CM, et al. SHANK3 mutations identified in autism lead to modification of dendritic spine morphology via an actin-dependent mechanism. Mol Psychiatry. 2012;17(1):71-84.

74. Han K, et al. SHANK3 overexpression causes manic-like behaviour with unique pharmacogenetic properties. Nature. 2013;503(7474):72-77.

75. Wang X, Xu Q, Bey AL, Lee Y, Jiang YH. Transcriptional and functional complexity of Shank3 provides a molecular framework to understand the phenotypic heterogeneity of SHANK3 causing autism and Shank 3 mutant mice. Mol Autism. 2014;5:30.

76. Duffney LJ, et al. Autism-like deficits in Shank3deficient mice are rescued by targeting actin regulators. Cell Rep. 2015;11(9):1400-1413.

77. Zhang $\mathrm{Q}$, et al. Impaired dendritic development and memory in Sorbs2 knock-out mice. J Neurosci. 2016;36(7):2247-2260.

78. Burguière E, Monteiro P, Mallet L, Feng G, Graybiel AM. Striatal circuits, habits, and implications for obsessive-compulsive disorder. Curr Opin Neurobiol. 2015;30:59-65.

79. Monteiro P, Feng G. Learning from animal models of obsessive-compulsive disorder. Biol Psychiatry. 2016;79(1):7-16.

80. Armbruster BN, Li X, Pausch MH, Herlitze S, Roth BL. Evolving the lock to fit the key to create a family of $G$ protein-coupled receptors potently activated by an inert ligand. Proc Natl Acad Sci U S A. 2007;104(12):5163-5168.

81. Pei Y, Dong S, Roth BL. Generation of designer receptors exclusively activated by designer drugs (DREADDs) using directed molecular evolution. Curr Protoc Neurosci. 2010; Chapter 4:Unit 4.33. 
82. Ferguson SM, Phillips PE, Roth BL, Wess J, Neumaier JF. Direct-pathway striatal neurons regulate the retention of decision-making strategies. J Neurosci. 2013;33(28):11668-11676.

83. Kravitz AV, et al. Regulation of parkinsonian motor behaviours by optogenetic control of basal ganglia circuitry. Nature. 2010;466(7306):622-626.

84. Lewis M, Kim SJ. The pathophysiology of restricted repetitive behavior. J Neurodev Disord. 2009; 1(2):114-132

85. Rothwell PE, et al. Autism-associated neuroligin-3 mutations commonly impair striatal circuits to boost repetitive behaviors. Cell. 2014; 158(1):198-212.

86. Espinosa F, Xuan Z, Liu S, Powell CM. Neuroligin 1 modulates striatal glutamatergic neurotransmission in a pathway and NMDAR subunit-specific manner. Front Synaptic Neurosci. 2015;7:11.

87. Doyle JP, et al. Application of a translational profiling approach for the comparative analysis of CNS cell types. Cell. 2008;135(4):749-762.

88. Heiman M, et al. A translational profiling approach for the molecular characterization of CNS cell types. Cell. 2008;135(4):738-748.

89. Jiang YH, Ehlers MD. Modeling autism by SHANK gene mutations in mice. Neuron. 2013; 78(1):8-27.

90. Huber KM, Gallagher SM, Warren ST, Bear MF. Altered synaptic plasticity in a mouse model of fragile X mental retardation. Proc Natl Acad Sci USA. 2002;99(11):7746-7750.
91. Bear MF, Huber KM, Warren ST. The mGluR theory of fragile $\mathrm{X}$ mental retardation. Trends Neurosci. 2004;27(7):370-377.

92. Auerbach BD, Osterweil EK, Bear MF. Mutations causing syndromic autism define an axis of synaptic pathophysiology. Nature. 2011;480(7375):63-68.

93. Chévere-Torres I, et al. Metabotropic glutamate receptor-dependent long-term depression is impaired due to elevated ERK signaling in the $\triangle \mathrm{RG}$ mouse model of tuberous sclerosis complex. Neurobiol Dis. 2012;45(3):1101-1110.

94. Peixoto RT, Wang W, Croney DM, Kozorovitskiy Y, Sabatini BL. Early hyperactivity and precocious maturation of corticostriatal circuits in Shank3B (-/-) mice. Nat Neurosci. 2016;19(5):716-724.

95. Saxena S, Rauch SL. Functional neuroimaging and the neuroanatomy of obsessivecompulsive disorder. Psychiatr Clin North Am. 2000;23(3):563-586.

96. Alexander GE, DeLong MR, Strick PL. Parallel organization of functionally segregated circuits linking basal ganglia and cortex. Annu Rev Neurosci. 1986;9:357-381.

97. Joel D, Doljansky J. Selective alleviation of compulsive lever-pressing in rats by $\mathrm{D} 1$, but not $\mathrm{D} 2$, blockade: possible implications for the involvement of $\mathrm{D} 1$ receptors in obsessive-compulsive disorder. Neuropsychopharmacology. 2003;28(1):77-85.

98. Presti MF, Mikes HM, Lewis MH. Selective blockade of spontaneous motor stereotypy via intrastriatal pharmacological manipulation. Pharmacol Biochem Behav. 2003;74(4):833-839.

99. Berridge KC, Aldridge JW, Houchard KR, Zhuang $\mathrm{X}$. Sequential super-stereotypy of an instinctive fixed action pattern in hyper-dopaminergic mutant mice: a model of obsessive compulsive disorder and Tourette's. BMC Biol. 2005;3:4.

100.Campbell KM, et al. OCD-Like behaviors caused by a neuropotentiating transgene targeted to cortical and limbic D1+ neurons. J Neurosci. 1999;19(12):5044-5053.

101. Kravitz AV, Tye LD, Kreitzer AC. Distinct roles for direct and indirect pathway striatal neurons in reinforcement. Nat Neurosci. 2012;15(6):816-818.

102. Garner JP. Perseveration and stereotypy: systems-level insights from clinical psychology. In: Mason G, Rushen J, eds. Stereotypic animal behaviour: fundamentals and applications to welfare. 2006; Wallingford: CAB International; 121-152.

103. Lewis MH, Presti MF, Lewis JB, Turner CA. The neurobiology of stereotypy I: Environmental complexity. In: Mason G, Rushen J, eds. Stereotypic animal behaviour: fundamentals and applications to welfare. 2006; Wallingford: CAB International; 190-226.

104. Yardeni T, Eckhaus M, Morris HD, Huizing M, Hoogstraten-Miller S. Retro-orbital injections in mice. Lab Anim (NY). 2011;40(5):155-160.

105.Van de Weerd HA, et al. Validation of a new system for the automatic registration of behaviour in mice and rats. Behav Processes. 2001;53(1-2):11-20. 